\title{
Influence of $N$-substituents of carbamoyl-stabilized azomethine ylides in 1,3-dipolar cycloadditions
}

\author{
Jiří Pospíšil, Martin Trávníček, and Milan Potáček* \\ Department of Organic Chemistry, Masaryk University of Brno, Kotlářská 2, \\ CZ-61137 Brno, Czech Republic \\ E-mail:potacek@,chemi.muni.cz
}

\begin{abstract}
Dedicated to Professor Fritz Sauter on the occasion of his birthday anniversary (received 03 Mar 01; accepted 04 Feb 02; published on the web12 Feb 02)
\end{abstract}

\begin{abstract}
Upon treatment with base $\mathrm{N}$-substituted carbamoylmethylphenanthridinium salts were converted into azomethine ylides. These intermediates were intercepted with symmetrically substituted dipolarophiles, and the stereochemistry of the cycloadducts has been found to be dependent on the substitution at the amide nitrogen atom.
\end{abstract}

Keywords: 1,3-Dipolar cycloaddition, azomethine ylides, $N$-substituted 2-bromoacetamides, phenanthridinium salts, pyrrolidino[1,2-f]phenanthridines

\section{Introduction}

Carbonyl-stabilized azomethine ylides (1,3-dipoles) derived from phenanthridinium salts are known to undergo versatile 1,3-dipolar cycloaddition reactions with a series of dipolarophiles (fumaronitrile, dimethyl fumarate, and dimethyl maleate). In this way five-membered rings can be fused to the phenanthridine scaffold to form pyrrolidino[1,2- $f$ phenanthridines. ${ }^{1-7}$ Such 1,3 dipolar cycloaddition reactions have been observed to proceed stereoselectively. ${ }^{1-5} \mathrm{We}$ are investigating the stereochemistry of pyrrolidino[1,2-f]phenanthridines resulting from these reactions. In addition, we have revealed that the reactivity of these azomethine ylides and hence the stereoselectivity of the cycloadducts strongly depend on the group attached to the carbonyl group stabilising the ylide. In the case of alkoxycarbonyl derivatives (esters) a high reactivity and a relatively low selectivity have been observed. ${ }^{1-5}$ Moreover, the cycloadducts obtained from these reactions are often accompanied by dehydrogenated products derived from them. On the other hand, a rather poor reactivity of aminocarbonyl derivatives (amides), was observed, ${ }^{6,7}$ the 
only reactive dipolarophile was fumaronitrile.

Natural products with phenanthridinium skeleton (phenanthridinium alkaloids) represent a broad group of biologically active compounds. ${ }^{8-12}$ Compounds with an adamantyl-substituted amino group are known for their biological activity as well. ${ }^{13-15}$ We wanted to study the influence of relatively bulky $\mathrm{N}$-substituents on the stereochemical course of the reaction and the formation of stereoisomers, and in addition, the preparation of products with the above mentioned structures was carried out in anticipation of their biological activity.

\section{Results and Discussion}

The reaction of phenanthridine $\mathbf{1}$ with $\mathrm{N}$-substituted $\alpha$-bromoacetamides 2 furnished the phenanthridinium bromides $\mathbf{3}$ as starting materials for the subsequent triethylamine-induced conversion into in situ formed azomethine ylides 4 .

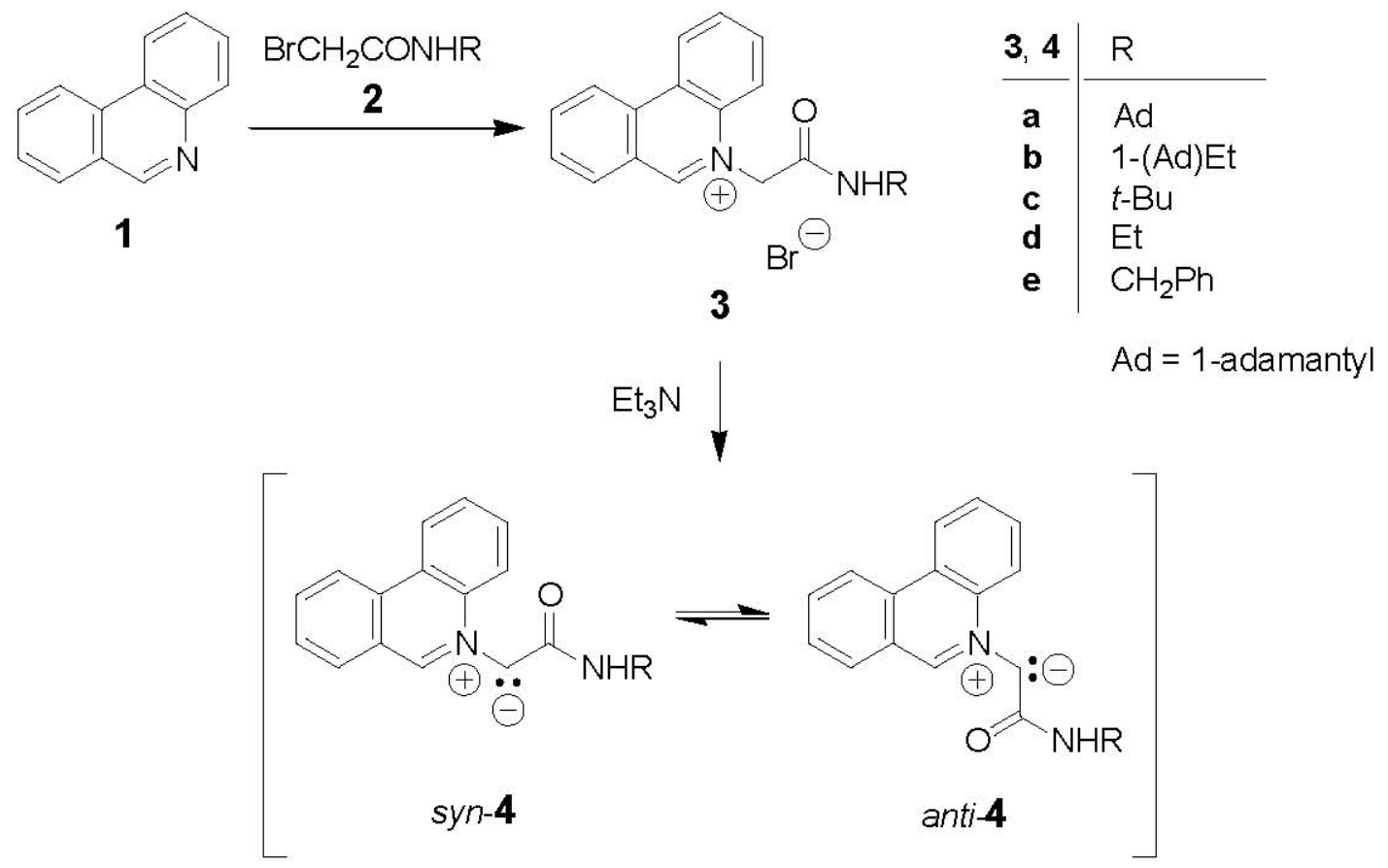

\section{Scheme 1}

The products obtained from azomethine ylides $\mathbf{4}$ reacting with symmetrically disubstituted dipolarophiles fumaronitrile $\mathbf{5 a}$, dimethyl fumarate $\mathbf{5 b}$, and dimethyl maleate $\mathbf{5 c}$ indicate the existence of two ylide conformers syn-4 and anti-4 (Scheme 1) as reflected by the stereochemistry of the cycloadducts 6, 7, and $\mathbf{1 1}$ (Scheme 2).

The cycloaddition reactions were carried out in an inert atmosphere. The cycloadducts formed in the reaction with the $E$-configurated dipolarophiles $\mathbf{5 a}$ and $\mathbf{5 b}$ were influenced by two 
factors, the temperature and the bulkiness of the substituent at the carbamoyl nitrogen atom. With a bulky group directly attached to the nitrogen atom (as in 3a, 3c) two stereoisomeric cycloadducts 6 and 7 were obtained. In case the substituent at the nitrogen atom is not sterically demanding (3d, 3e) or if there is a spacer between the bulky group and the nitrogen atom (3b) only one stereoisomer was formed (Table 1).
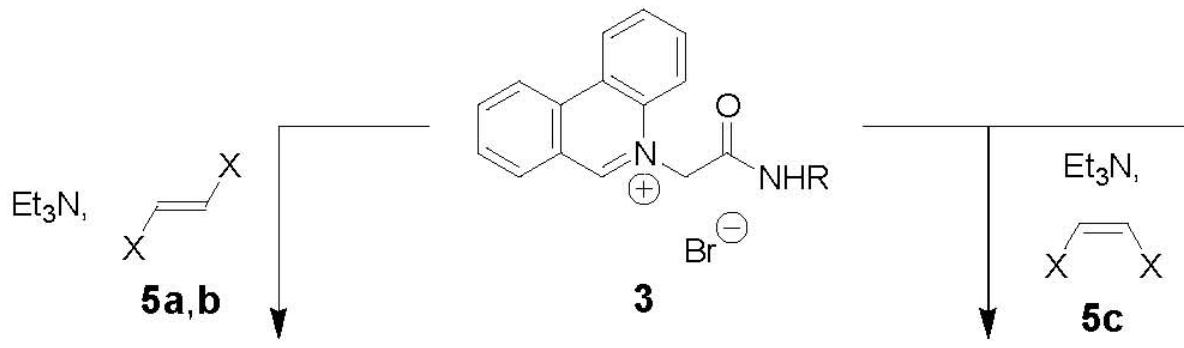

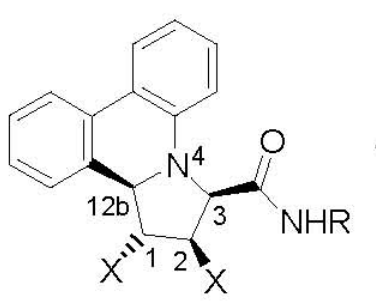

6

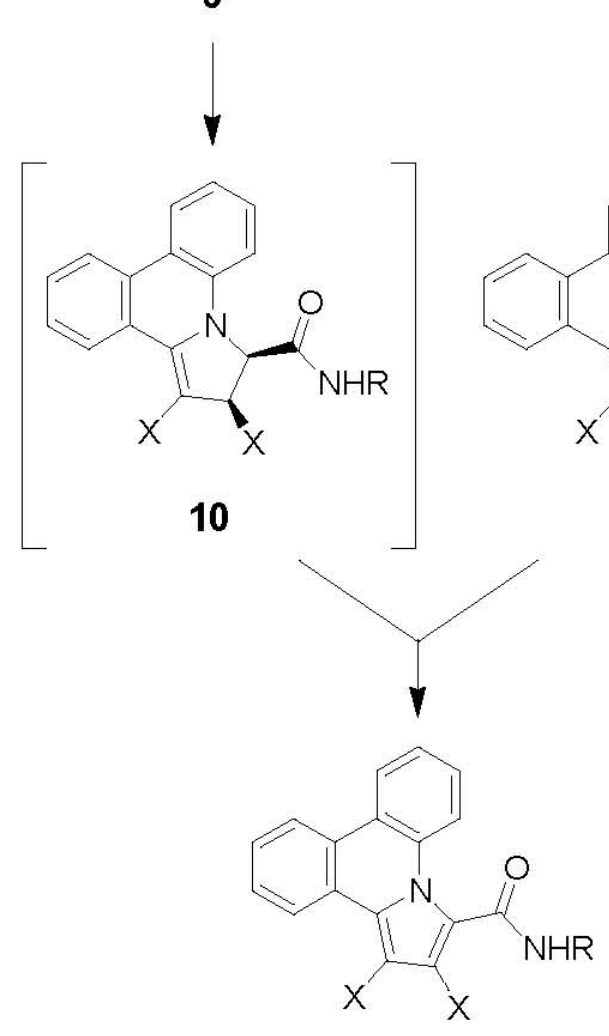

9<smiles>[R]NC(=O)[C@H]1[C@@H]([X])[C@@H]([X])[C@@H]2c3ccccc3-c3ccccc3N12</smiles>

7

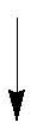<smiles>[R]NC(=O)[C@H]1C([X])[C@@H]([X])[C@@H]2c3ccccc3-c3ccccc3N12</smiles>

$11 b c$

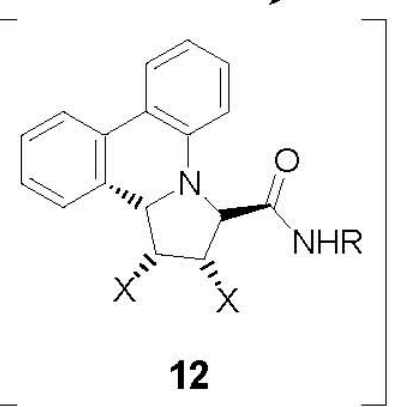

12

\begin{tabular}{|c|c|c|}
\hline products & $\mathrm{R}$ & $x$ \\
\hline $\begin{array}{l}\text { 6aa,7aa, 9aa } \\
6 a b, 7 a b ; 8 a c\end{array}$ & $\begin{array}{l}\mathrm{Ad} \\
\mathrm{Ad}\end{array}$ & $\begin{array}{l}\mathrm{CN} \\
\mathrm{CO}_{2} \mathrm{Me}\end{array}$ \\
\hline $\begin{array}{l}6 b a \\
6 b b ; 11 b c\end{array}$ & $\begin{array}{l}\text { 1-(Ad)Et } \\
\text { 1-(Ad)Et }\end{array}$ & $\begin{array}{l}\mathrm{CN} \\
\mathrm{CO}_{2} \mathrm{Me}\end{array}$ \\
\hline $\begin{array}{l}6 \mathrm{ca}, 7 \mathrm{ca}, 8 \mathrm{ca}, 9 \mathrm{ca} \\
6 \mathrm{cb}, 7 \mathrm{cb} ; 8 \mathrm{cc}\end{array}$ & $\begin{array}{l}t \text {-Bu } \\
t \text {-Bu }\end{array}$ & $\begin{array}{l}\mathrm{CN}^{2} \\
\mathrm{CO}_{2} \mathrm{Me}\end{array}$ \\
\hline $\begin{array}{l}6 \mathrm{da} \\
6 \mathrm{db} ; 8 \mathrm{dc}\end{array}$ & & $\begin{array}{l}\mathrm{CN} \\
\mathrm{CO}_{2} \mathrm{Me}\end{array}$ \\
\hline 6ea & $\mathrm{CH}_{2} \mathrm{Ph}$ & $\mathrm{CN}$ \\
\hline $8 \mathrm{ec}$ & $\mathrm{CH}_{2} \mathrm{Ph}$ & $\mathrm{CO}_{2} \mathrm{Me}$ \\
\hline
\end{tabular}

Scheme 2 
Table 1. Products 6-9 from the reaction of salts $\mathbf{3}$ with fumaronitrile $\mathbf{5 a}$ or dimethyl fumarate $\mathbf{5 b}$ in the presence of triethylamine as determined by the reaction conditions: inert atmosphere; solvent (dry) and reaction temperature (Methods 1, 2 and 3), and reaction time.

\begin{tabular}{|c|c|c|c|c|c|c|c|c|}
\hline Entry & 3 & 5 & Solvent & Temp. & Method & Time & Product(s) (ratio) & Yield [\%] \\
\hline 1 & $3 \mathbf{a}$ & $5 \mathbf{a}$ & $\mathrm{CH}_{2} \mathrm{Cl}_{2}$ & ambient & 1 & 2 days & - & $<5$ \\
\hline 2 & $\mathbf{3 a}$ & $5 \mathbf{a}$ & $\mathrm{CH}_{2} \mathrm{Cl}_{2}$ & ambient & 1 & 2 months & 6aa, $7 \mathbf{a a}(79: 21)$ & 49 \\
\hline 3 & $\mathbf{3 a}$ & $5 \mathbf{a}$ & $\mathrm{CH}_{2} \mathrm{Cl}_{2}$ & reflux & 2 & 2 days & 6aa, 7aa $(79: 21)$ & 95 \\
\hline 4 & $\mathbf{3 a}$ & $5 \mathbf{a}$ & $\mathrm{CHCl}_{3}$ & reflux & 3 & 2 days & 9aa & 94 \\
\hline 5 & $\mathbf{3 a}$ & $5 b$ & $\mathrm{CH}_{2} \mathrm{Cl}_{2}$ & ambient & 1 & 2 months & $\mathbf{6 a b}, 7 \mathbf{a b}(56: 44)$ & 47 \\
\hline 6 & $\mathbf{3 a}$ & $5 b$ & $\mathrm{CHCl}_{3}$ & reflux & 3 & 2 days & 6ab, 7ab (91:9) & 89 \\
\hline 7 & $3 \mathbf{b}$ & $5 \mathbf{a}$ & $\mathrm{CH}_{2} \mathrm{Cl}_{2}$ & ambient & 1 & 2 days & $6 b a$ & 52 \\
\hline 8 & $3 \mathbf{b}$ & $5 \mathbf{a}$ & $\mathrm{CH}_{2} \mathrm{Cl}_{2}$ & reflux & 2 & 2 days & $6 \mathbf{b a}$ & 26 \\
\hline 9 & $3 \mathbf{b}$ & $5 \mathbf{b}$ & $\mathrm{CH}_{2} \mathrm{Cl}_{2}$ & ambient & 1 & 2 days & $6 \mathbf{b b}$ & 48 \\
\hline 10 & $3 c$ & $5 \mathbf{a}$ & $\mathrm{CH}_{2} \mathrm{Cl}_{2}$ & ambient & 1 & 2 days & - & $<5$ \\
\hline 11 & $3 c$ & $5 \mathbf{a}$ & $\mathrm{CH}_{2} \mathrm{Cl}_{2}$ & ambient & 1 & 2 months & 6ca, 7ca $(67: 33)$ & 48 \\
\hline 12 & $3 c$ & $5 \mathbf{a}$ & $\mathrm{CH}_{2} \mathrm{Cl}_{2}$ & reflux & 2 & 2 days & 6ca, 7ca $(67: 33)$ & 80 \\
\hline 13 & $3 c$ & $5 \mathbf{a}$ & $\mathrm{CHCl}_{3}$ & reflux & 3 & 2 days & 8ca, 9ca $(71: 29)$ & 62 \\
\hline 14 & $3 c$ & $5 \mathbf{b}$ & $\mathrm{CH}_{2} \mathrm{Cl}_{2}$ & ambient & 1 & 2 months & 6cb, 7cb $(54: 46)$ & 47 \\
\hline 15 & $3 c$ & $5 \mathbf{b}$ & $\mathrm{CHCl}_{3}$ & reflux & 3 & 2 days & $6 \mathrm{cb}$ & 36 \\
\hline 16 & $3 d$ & $5 \mathbf{a}$ & $\mathrm{CH}_{2} \mathrm{Cl}_{2}$ & ambient & 1 & 2 days & $6 \mathrm{da}$ & 52 \\
\hline 17 & $3 d$ & $5 \mathbf{a}$ & $\mathrm{CH}_{2} \mathrm{Cl}_{2}$ & reflux & 2 & 2 days & $6 \mathrm{da}$ & 73 \\
\hline 18 & $3 d$ & $5 \mathbf{b}$ & $\mathrm{CH}_{2} \mathrm{Cl}_{2}$ & ambient & 1 & 2 days & $6 \mathrm{db}$ & 45 \\
\hline 19 & $3 \mathbf{e}$ & $5 \mathbf{a}$ & $\mathrm{CH}_{2} \mathrm{Cl}_{2}$ & ambient & 1 & 2 days & $6 \mathrm{ea}$ & 49 \\
\hline 20 & $3 \mathbf{e}$ & $5 \mathbf{a}$ & $\mathrm{CH}_{2} \mathrm{Cl}_{2}$ & reflux & 2 & 2 days & 6ea & 75 \\
\hline
\end{tabular}

The yields of cycloadducts $\mathbf{6}$ and $\mathbf{7}$ isolated from the reaction of salts $\mathbf{3}$ with fumaronitrile $\mathbf{5 a}$ vary sensibly with temperature at a reaction time of two days (Table 1: Methods 1 and 2, entries 2 and $3 ; 7$ and $8 ; 11$ and 12;16 and 17;19 and 20); the slightly increased temperature of boiling dichloromethane (Method 2) significantly increased the cycloadduct yields (except for $\mathbf{6 b a}$ ). The reactions of salts $\mathbf{3 a}$ and $\mathbf{3 c}(\mathrm{R}=$ bulky $\mathrm{N}$-substituents) with fumaronitrile $\mathbf{5 a}$ at ambient temperature (Method 1) barely provided any cycloaddition products after two days (Table 1, entries 1 and 10) but required a rather prolonged reaction period of two months to yield cycloadducts as a mixture of stereoisomers 6 and 7 (Table 1, entries 2 and 11). When the cycloaddition reactions of 3a and 3c with 5a were carried out in boiling chloroform (Method 3), dehydrogenated products 8 and/or 9 were obtained instead of the expected cycloadducts 6 and 7 (Table 1, entries 4 and 13). Remarkably, no partially dehydrogenated products $\mathbf{1 0}$ (derived from cycloadducts 6) have been found in these reactions (Method 3) though cycloadducts 6 are the 
major products at lower reaction temperatures (Methods 1 and 2, vide supra). Obviously, dehydrogenation of $\mathbf{1 0}$ (cis-2,3-dihydro structure) and the conversion into the completely unsaturated products 9 occurs faster than the further dehydrogenation of $\mathbf{8}$ (the trans-2,3-dihydro isomer) into 9.

The reaction of dimethyl fumarate $\mathbf{5 b}$ with $\mathbf{3 a}$ and $\mathbf{3 c}(\mathrm{R}=$ bulky $\mathrm{N}$-substituents) at ambient temperature (Method 1) and after a prolonged reaction time of two months furnished the respective cycloadducts 6 and 7 (Table 1, entries 5, 14); at elevated reaction temperature (Method 3) the formation of cycloadducts 6 was strongly favored or was the exclusive product (Table 1, entries 6 and 15, respectively). The salts $\mathbf{3 b}$ and $\mathbf{3 d}$ (with sterically less demanding Nsubstituents) at ambient reaction temperature (Method 1) gave rise to the formation of cycloadducts 6 exclusively (Table 1, entries 9, 18).

Table 2. Temperature-dependent formation of products 8 and 11 bc from the reaction of salts 3 with dimethyl maleate $\mathbf{5 c}$ (in dichloromethane in inert atmosphere after 2 days)

\begin{tabular}{ccccc}
\hline 3 & Temperature & Method & Product & Yield [\%] \\
\hline 3a & ambient & 1 & - & $<5$ \\
3a & reflux & 2 & $\mathbf{8 a c}$ & 34 \\
3b & ambient & 1 & $\mathbf{1 1 b c}$ & 47 \\
3b & reflux & 2 & $\mathbf{1 1 b c}$ & 15 \\
3c & ambient & 1 & - & $<5$ \\
3c & reflux & 2 & $\mathbf{8 c c}$ & 31 \\
3d & ambient & 1 & $\mathbf{8 d c}$ & 45 \\
3d & reflux & 2 & $\mathbf{8 d c}$ & 20 \\
3e & ambient & 1 & $\mathbf{8 e c}$ & 48 \\
3e & reflux & 2 & $\mathbf{8 e c}$ & 25 \\
\hline
\end{tabular}

The primarily formed cycloadducts resulting from the reaction of salts $\mathbf{3}$ with dimethyl maleate 5c appear to be very sensitive to dehydrogenation. With the exception of salt $\mathbf{3 b}$ giving rise to the formation of cycloadduct $11 b c$ (reflecting the anti-conformation of the azomethine ylide intermediate $\mathbf{4 b}$ ) all other salts 3a,c-e afforded partially dehydrogenated products 8ac, 8cc, 8dc, and 8ec, respectively (Table 2). Apparently, these products are formed via structure $\mathbf{1 2}$ featuring cis-orientation of $\mathrm{H}-1$ and $\mathrm{H}-12 \mathrm{~b}^{1,2}$ very easily undergo dehydrogenation to the isolated products 8ac, 8cc, 8dc, and 8ec. Product 11 bc with trans configuration at $\mathrm{C}-1, \mathrm{C} 12 \mathrm{~b}$ resists dehydrogenation thus enabling its isolation. Obviously, the bulky groups $\mathrm{R}$ of the starting materials 3a and 3c retard the cycloaddition reaction with dimethyl maleate $\mathbf{5 c}$ at ambient temperature (Method 1), and a slightly higher temperature (Method 2) is required for the reaction to proceed. On the other hand, the reaction of the starting materials $\mathbf{3 b}$ and $\mathbf{3 d}$ (with sterically 
less demanding groups $\mathrm{R}$ attached to the amide nitrogen atom) reacted already at ambient temperature (Method 1) affording products 8. However, a slightly higher reaction temperature (Method 2) caused a significant decrease in product yield probably because of decomposition.

Table 3. Experimental coupling constants $\left({ }^{3} J_{\text {exp }}\right)$ and coupling constants calculated $\left({ }^{3} J_{\text {calc }}\right)$ with the Karplus equation using dihedral angles $(\Phi)$ obtained by the semi-empirical AM1 method (SPARTAN program) for cycloadducts 6, 7 and $\mathbf{1 1 b c}$

\begin{tabular}{|c|c|c|c|c|c|c|c|c|c|}
\hline \multicolumn{2}{|c|}{ Product } & \multicolumn{3}{|c|}{ H-12b-H-1 } & \multicolumn{3}{|c|}{$\mathrm{H}-1-\mathrm{H}-2$} & \multicolumn{2}{|c|}{$\mathrm{H}-2-\mathrm{H}-3$} \\
\hline${ }^{3} J_{\exp }$ & $\Phi\left[^{\circ}\right]$ & ${ }^{3} J_{\text {calc }}$ & ${ }^{3} J_{\exp }$ & & & ${ }^{3} J_{\text {calc }}$ & ${ }^{3} J_{\exp }$ & $\Phi\left[^{\circ}\right]$ & ${ }^{3} J_{\text {calc }}$ \\
\hline$[\mathrm{Hz}]$ & (AM1) & {$[\mathrm{Hz}]$} & {$[\mathrm{Hz}]$} & & M1) & {$[\mathrm{Hz}]$} & {$[\mathrm{Hz}]$} & (AM1) & {$[\mathrm{Hz}]$} \\
\hline 6aa & 4.7 & 136.2 & 4.8 & 9.7 & 172.3 & 9.1 & 10.0 & 0.5 & 8.2 \\
\hline $7 \mathbf{a a}$ & 8.3 & 2.0 & 7.9 & 0.0 & 102.4 & 0.2 & 4.2 & 134.2 & 4.2 \\
\hline $6 \mathrm{ba}$ & 4.6 & 134.9 & 4.6 & 9.9 & 179.5 & 9.2 & 10.0 & 1.1 & 8.2 \\
\hline $6 \mathrm{ca}$ & 4.7 & 135.6 & 4.7 & 9.9 & 176.2 & 9.2 & 9.9 & 2.1 & 7.9 \\
\hline $7 \mathrm{ca}$ & 8.7 & 1.4 & 8.2 & 0.0 & 99.2 & 0.0 & 3.5 & 128.4 & 6.5 \\
\hline 6da & 4.7 & 135.9 & 4.7 & 9.9 & 177.3 & 9.2 & 9.9 & 1.5 & 8.2 \\
\hline 6еa & 4.0 & 131.9 & 4.1 & 9.9 & 176.4 & 9.2 & 9.9 & 2.7 & 7.9 \\
\hline 6ab & 5.2 & 139.5 & 5.3 & 9.5 & 169.3 & 9.1 & 9.5 & 3.2 & 7.8 \\
\hline $7 \mathbf{a b}$ & 9.1 & 1.3 & 8.1 & 0.0 & 100.2 & 0.1 & 5.7 & 142.6 & 5.8 \\
\hline $6 b b$ & 5.0 & 137.6 & 5.0 & 9.4 & 168.4 & 9.0 & 9.4 & 3.0 & 7.8 \\
\hline $6 \mathrm{cb}$ & 5.3 & 140.2 & 5.4 & 9.3 & 168.1 & 9.0 & 9.3 & 4.1 & 7.7 \\
\hline $7 \mathrm{cb}$ & 9.4 & 1.1 & 8.2 & 0.0 & 99.4 & 0.0 & 5.7 & 141.8 & 5.7 \\
\hline $6 \mathrm{db}$ & 5.2 & 139.8 & 5.2 & 9.4 & 169.7 & 9.1 & 9.4 & 2.9 & 7.8 \\
\hline $11 b c$ & 4.7 & 136.4 & 4.7 & 0.0 & 87.2 & 0.2 & 9.4 & 3.2 & 7.8 \\
\hline
\end{tabular}

The assignment of the relative configuration at $\mathrm{C}-1, \mathrm{C}-2, \mathrm{C}-3$, and $\mathrm{C}-12 \mathrm{~b}$ is based on the coupling constants ${ }^{3} J$ under the premise that the reaction proceeds with preservation of dipolarophile configuration. The vicinal coupling constants ${ }^{3} J_{1,12 \mathrm{~b}}$ and ${ }^{3} J_{2,3}$ have been found in the following ranges: ${ }^{3} J_{\text {cis }}=8.5-11.5 \mathrm{~Hz}$ and ${ }^{3} J_{\text {trans }}=2.5-5.5 \mathrm{~Hz}{ }^{1-6}$ However, these values ${ }^{3} J_{\text {cis }}$ and ${ }^{3} J_{\text {trans }}$ are not applicable to ${ }^{3} J_{1,2} \cdot{ }^{16}$ The values reflect conformation of the five-membered ring. Unfortunately, we were not able to obtain a suitable crystal for X-ray analysis to support the assignments; therefore, we tried to support the structure with data from quantum chemical calculations. The semi-empirical AM1 method (SPARTAN program) was employed for the calculation and optimization of the space arrangement of adducts $\mathbf{6}, \mathbf{7}$, and $\mathbf{1 1 b}$. Based on the dihedral angles of the hydrogen atoms attached to the five-membered ring the coupling constants were calculated by application of the Karplus equation; ${ }^{17}$ the calculated values together with the 
experimental coupling constants are listed in Table 3. All calculated results are in a good agreement with experimental data and the structure elucidation by $2 \mathrm{D}$ NMR experiments. The value of the coupling constant depends on the conformation of the five-membered ring resulting from the cycloaddition reaction, and therefore, ${ }^{3} J_{1,2}$ values differ from those found for ${ }^{3} J_{1,12 b}$ and ${ }^{3} J_{2,3}$. With the dihedral angle H-1-C-1-C-2-H-2 close to $180^{\circ}{ }^{3} J_{1,2}$ is relatively large (compounds 6); when the dihedral angle approaches $90^{\circ}$, the coupling constant ${ }^{3} J_{1,2}$ is negligible (compounds 7). The experimental coupling constants are in very good agreement with the theoretical values obtained on the basis of the calculated dihedral angle. Differences between experimental and calculated ${ }^{3} J$ values are observed only in those cases where the dihedral angle between neighboring hydrogen atoms is very close to zero (Table 3 ) and C-2 and C-3 are bearing different substituents. This causes divergent values of ${ }^{3} J_{2,3}$ reflecting the fact that the Karplus equation does not consider electronic effect of adjacent groups.

The ${ }^{1} \mathrm{H}$ NMR spectra of compounds $\mathbf{6 b a}$ and $\mathbf{1 1 b c}$ exhibit duplicate sets of pyrrolidine ring signals owing to mixtures of diastereomers because the starting material 3a was a racemic mixture; integration of the proton signals revealed a 1:1 ratio of the diastereomers.

Some adamantly-substituted compounds have been described earlier; ${ }^{7}$ the experiments were repeated, the product structures were reinvestigated, and the results are included in order to put them into perspective with those of the other products described herein.

\section{Experimental Section}

General Procedures. Melting points were measured on a Kofler hot stage VEB Wägetechnik Rapido 79/2106. IR spectra were recorded as $\mathrm{KBr}$ pellets on a FTIR ATI MATTSON spectrophotometer. NMR spectra were recorded on a Bruker Avance DPX 300 apparatus with working frequency $300 \mathrm{MHz}$ for ${ }^{1} \mathrm{H}$ and $75 \mathrm{MHz}$ for ${ }^{13} \mathrm{C}$ in $\mathrm{CDCl}_{3}$ or DMSO- $d_{6}$ solution with TMS as an internal standard. Mass spectra were recorded on a FISONS INSTRUMENTS TRIO 1000 spectrometer in positive mode with EI ionization $(20 \mathrm{eV})$. TLC was carried out on commercial silica plates Silufol Kavalier, Czech Republic. Column chromatography was carried out on Merck silica (63-100 $\mu \mathrm{m}$ ). Dichloromethane (product of Onex, Czech Republic) was dried over $\mathrm{CaH}_{2}$ and distilled from it. Triethylamine was dried over $\mathrm{KOH}$ and rectified through a column with $\mathrm{BaO}$.

\section{General method for the preparation of $\mathrm{N}$-substituted 2-bromoacetamides 2a-e}

To a solution of amine $(90.98 \mathrm{mmol})$ in dry dichloromethane $(50 \mathrm{~mL})$ cooled to -20 to $-30{ }^{\circ} \mathrm{C}$ bromoacetyl bromide $(9.18 \mathrm{~g}, 45.49 \mathrm{mmol})$ was added dropwise under stirring and cooling of the strong exothermic reaction while a white precipitate was formed. The reaction temperature during the addition of bromoacetyl bromide should not exceed $-10{ }^{\circ} \mathrm{C}$. After all bromoacetyl 
bromide was added stirring was continued for another $20 \mathrm{~min}$ at the same temperature. Finally, the reaction mixture was filtered, the filtrate was evaporated in vacuo, and the residue was crystallized from an appropriate solvent.

$\mathbf{N}$-(1-Adamantyl)-2-bromoacetamide ${ }^{7}$ (2a). Colorless crystals (from benzene) $(7.8 \mathrm{~g}, 63 \%), \mathrm{mp}$ 124-126 ${ }^{\circ} \mathrm{C}$; IR (KBr): $3266(\mathrm{NH}), 3081,2904,2856,1656(\mathrm{C}=\mathrm{O}), 1563(\mathrm{NH}), 1454,1402$, 1305, 1211, 1093, 998, 914, 750, 696, $565 \mathrm{~cm}^{-1} ;{ }^{1} \mathrm{H} \mathrm{NMR}\left(\mathrm{CDCl}_{3}\right): \delta 1.69\left(\mathrm{~s}, 6 \mathrm{H}, 3 \mathrm{CH}_{2} \mathrm{Ad}\right)$, 2.02 (s, 6H, $\left.3 \mathrm{CH}_{2} \mathrm{Ad}\right), 2.10$ (s, 3H, $3 \mathrm{CH}_{\mathrm{Ad}}$ ), 3.77 (s, $\left.2 \mathrm{H}, \mathrm{CH}_{2} \mathrm{Br}\right), 6.15$ (s, 1H, NH); ${ }^{13} \mathrm{C}$ NMR $\left(\mathrm{CDCl}_{3}\right): \delta 30.01\left(\mathrm{CH}_{\mathrm{Ad}}\right), 36.35\left(\mathrm{CH}_{2} \mathrm{Ad}\right), 41.31\left(\mathrm{CH}_{2} \mathrm{Ad}\right), 52.68\left(\mathrm{CH}_{2} \mathrm{Br}\right), 164.24(\mathrm{C}=\mathrm{O})$. Anal. Calcd. for $\mathrm{C}_{12} \mathrm{H}_{18} \mathrm{BrNO}$ (272.18): C, 52.95; H, 6.67; N, 5.15. Found: C, 52.60; H, 6.70; N, 5.13. $N$-[(1RS)-1-(1-Adamantyl)ethyl]-2-bromoacetamide ${ }^{7}$ (2b). Colorless crystals (from benzene) (8.19 g, 60\%), mp 128-129 ${ }^{\circ} \mathrm{C}$; IR (KBr): 3294 (NH), 3044, 2902, 2847, 1647 (C=O), 1559 (amide II), 1449, 1383, 1160, 722, $555 \mathrm{~cm}^{-1} ;{ }^{1} \mathrm{H} \mathrm{NMR}\left(\mathrm{CDCl}_{3}\right): \delta 1.04\left(\mathrm{~d}, 3 \mathrm{H}, J=6.9 \mathrm{~Hz}, \mathrm{CH}_{3}\right)$, 1.36 (q, $J=6.9 \mathrm{~Hz}, 1 \mathrm{H}, \mathrm{HC}-\mathrm{N}), 1.53-1.62$ (m, 12H, $6 \mathrm{CH}_{2 \mathrm{Ad}}$ ), 1.99 (br, 3H, $3 \mathrm{CH}_{\mathrm{Ad}}$ ), 3.63 (br s, $1 \mathrm{H}, \mathrm{NH}), 3.89\left(\mathrm{~s}, 2 \mathrm{H}, \mathrm{CH}_{2} \mathrm{Br}\right) ;{ }^{13} \mathrm{C} \mathrm{NMR}\left(\mathrm{CDCl}_{3}\right): \delta 14.12\left(\mathrm{CH}_{3}\right), 28.13\left(\mathrm{CH}_{\mathrm{Ad}}\right), 34.70\left(\mathrm{CH}_{2} \mathrm{Br}\right)$. $36.86\left(\mathrm{CH}_{2} \mathrm{Ad}\right), 38.21\left(\mathrm{CH}_{2} \mathrm{Ad}\right), 53.90(\mathrm{CH}-\mathrm{N}), 164.46(\mathrm{C}=\mathrm{O})$. Anal. Calcd. for $\mathrm{C}_{14} \mathrm{H}_{22} \mathrm{BrNO}$ (300.23): C, 56.01; H, 7.39; N, 4.67. Found: C, 55.99; H, 7.30; N, 4.55.

$\mathrm{N}$-(tert-Butyl)-2-bromoacetamide (2c). Colorless crystals (from benzene) (5.29 g, 60\%), mp 111-112 ${ }^{\circ} \mathrm{C}$; IR (KBr): $3309(\mathrm{NH}), 3074,2973,2888,1679(\mathrm{C}=\mathrm{O}), 1551(\mathrm{NH}), 1454,1402$, 1305, 1212, 1093, 998, 933, 750, 696, $573 \mathrm{~cm}^{-1} ;{ }^{1} \mathrm{H}$ NMR $\left(\mathrm{CDCl}_{3}\right): \delta 1.38\left[\mathrm{~s}, 9 \mathrm{H}, 3 \mathrm{CH}_{3}\right), 1.52$ (s, 1H, NH), $3.79\left(\mathrm{~s}, 2 \mathrm{H}, \mathrm{CH}_{2}\right) ;{ }^{13} \mathrm{C} \mathrm{NMR}\left(\mathrm{CDCl}_{3}\right) \delta: 28.38\left(\mathrm{CH}_{3}\right), 29.82\left(\mathrm{CH}_{2}\right), 164.43(\mathrm{C}=\mathrm{O})$. Anal. Calcd. for $\mathrm{C}_{6} \mathrm{H}_{12} \mathrm{BrNO}$ (194.07): C, 37.13; H, 6.23; N, 7.22. Found: C, 36.80; H, 6.00; N, 7.32 .

$N$-Ethyl-2-bromoacetamide (2d). Colorless oil (4.22 g, 56\%), bp $80{ }^{\circ} \mathrm{C} / 5 \mathrm{~mm} \mathrm{Hg}$; IR (KBr): 3278 (NH), 3073, 2976, 2878, $1657(\mathrm{C}=\mathrm{O}), 1551(\mathrm{NH}), 1443,1308,1208,1151,796,549 \mathrm{~cm}^{-1}$; ${ }^{1} \mathrm{H} \mathrm{NMR}\left(\mathrm{CDCl}_{3}\right): \delta 2.78\left(\mathrm{t}, 3 \mathrm{H}, \mathrm{CH}_{3}\right), 3.29\left(\mathrm{q}, 2 \mathrm{H}, \mathrm{CH}_{2} \mathrm{~N}\right), 3.93\left(\mathrm{~s}, 2 \mathrm{H}, \mathrm{CH}_{2} \mathrm{Br}\right), 7.78(\mathrm{~s}, 1 \mathrm{H}$, $\mathrm{NH}) ;{ }^{13} \mathrm{C}$ NMR $\left(\mathrm{CDCl}_{3}\right): \delta 14.17\left(\mathrm{CH}_{3}\right), 28.10\left(\mathrm{CH}_{2} \mathrm{~N}\right), 35.64\left(\mathrm{CH}_{2} \mathrm{Br}\right), 167,26(\mathrm{C}=\mathrm{O})$. Anal. Calcd. for $\mathrm{C}_{4} \mathrm{H}_{8} \mathrm{BrNO}$ (166.01): C, 28.94; H, 4.86; N, 8.44. Found: C, 28.67; H, 4.57; N, 8.20.

$N$-Benzyl-2-bromoacetamide ${ }^{7}$ (2e). Colorless oil (5.49 g, 53\%), bp $125-127{ }^{\circ} \mathrm{C}$; IR (KBr): $3277(\mathrm{NH})$, 3069, 3016, 2952, 2853, $1646(\mathrm{C}=\mathrm{O}), 1553(\mathrm{NH}), 1453,1420,1322,1210,1145$, 1059, 1005, 895, 748, 698, $495 \mathrm{~cm}^{-1} ;{ }^{1} \mathrm{H} \mathrm{NMR}\left(\mathrm{CDCl}_{3}\right): \delta 4.43\left(\mathrm{~d}, 2 \mathrm{H}, \mathrm{CH}_{2} \mathrm{~N}\right), 3.86(\mathrm{~s}, 2 \mathrm{H}$,

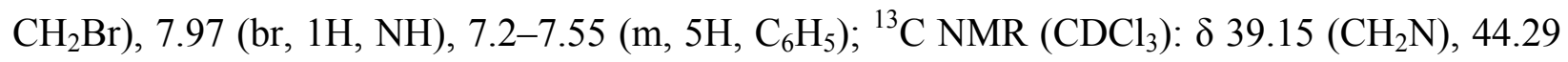
$\left(\mathrm{CH}_{2} \mathrm{Br}\right), 127.84-137.49\left(\mathrm{C}_{6} \mathrm{H}_{5}\right), 165.81(\mathrm{C}=\mathrm{O})$. Anal. Calcd. for $\mathrm{C}_{9} \mathrm{H}_{10} \mathrm{BrNO}$ (228.08): $\mathrm{C}, 47.39$; H, 4.42; N, 6.14. Found: C, 47.00; H, 4.22; N, 5.89.

\section{5-[2-(Alkylamino)-2-oxoethyl]phenantridinium bromides 3. General procedure}

To a hot solution $\left(65^{\circ} \mathrm{C}\right)$ of corresponding bromoacetamide $\mathbf{2 a}-\mathbf{2 e}(14.7 \mathrm{mmol})$ in acetonitrile $(50 \mathrm{~mL})$ a solution of phenanthridine $1(2.63 \mathrm{~g}, 14.7 \mathrm{mmol})$ in chloroform was added, and the mixture was refluxed for 3 days. A yellowish solid precipitated, and the solution turned orange. 
The reaction mixture was concentrated in vacuo, the precipitate was collected, washed with chloroform and diethyl ether, and air-dried at room temperature.

5-[2-(1-Adamantylamino)-2-oxoethyl]phenantridinium bromide ${ }^{7}$ (3a). Green-grey crystals (from toluene) (4.64 g, 70\%); 228-230 ${ }^{\circ} \mathrm{C}$; IR (KBr): $3187(\mathrm{NH}), 3037\left(\mathrm{CH}_{\text {arom }}\right), 2904,2848$, $1683(\mathrm{C}=\mathrm{O}), 1625\left(\mathrm{C}=\mathrm{N}^{+}\right), 1552,1450,1359,1259,1089,750,713 \mathrm{~cm}^{-1}$; ${ }^{1} \mathrm{H}$ NMR (DMSO- $\left.d_{6}\right)$ : $\delta$ 1.61-2.01 (m, 12H, $\left.6 \mathrm{CH}_{2} \mathrm{Ad}\right), 3.30\left(\mathrm{br}, 3 \mathrm{H}, 3 \mathrm{CH}_{\mathrm{Ad}}\right), 5.94\left(\mathrm{~s}, 2 \mathrm{H}, \mathrm{CH}_{2}\right), 8.10-9.19(\mathrm{~m}, 9 \mathrm{H}$, $\left.\mathrm{CH}_{\text {arom }}\right), 10.40(\mathrm{~s}, 1 \mathrm{H}, \mathrm{NH}) ;{ }^{13} \mathrm{C} \mathrm{NMR}\left(\mathrm{DMSO}-d_{6}\right): \delta 30.72\left(\mathrm{CH}_{\mathrm{Ad}}\right), 37.79\left(\mathrm{CH}_{2} \mathrm{Ad}\right), 42.75\left(\mathrm{CH}_{2}\right.$ Ad), $54.03\left(\mathrm{C}_{\mathrm{Ad}}\right), 120.96-140.47\left(\mathrm{CH}_{\text {arom }}\right.$ and $\left.\mathrm{C}_{\text {arom }}\right), 155.35\left(\mathrm{C}=\mathrm{N}^{+}\right), 164.52(\mathrm{C}=\mathrm{O})$. Anal. Calcd. for $\mathrm{C}_{25} \mathrm{H}_{27} \mathrm{BrN}_{2} \mathrm{O}$ (451.40): C, 66.52; H, 6.03; N, 6.21. Found: C, 66.22; H, 6.00; N, 5.93.

5-[2-[(1RS)-1-(1-Adamantyl)-1-ethylamino]-2-oxoethyl]phenantridinium bromide $^{7}$ (3b). Slightly green grey crystals (from toluene) (4.72 g, 67\%), mp 175-177 ${ }^{\circ} \mathrm{C}$; IR (KBr): $3416(\mathrm{NH})$, 3206, 2901, 2847, $1683(\mathrm{C}=\mathrm{O}), 1626\left(\mathrm{C}=\mathrm{N}^{+}\right), 1451,758.5 \mathrm{~cm}^{-1} ;{ }^{1} \mathrm{H}$ NMR (DMSO- $\left.d_{6}\right): \delta 1.04(\mathrm{~d}$, $\left.3 \mathrm{H}, J=6.9 \mathrm{~Hz}, \mathrm{CH}_{3}\right), 1.36$ (q, 1H, $\left.J=6.9, \mathrm{HC}-\mathrm{N}\right), 1.53-1.62\left(\mathrm{~m}, 12 \mathrm{H}, 6 \mathrm{CH}_{2} \mathrm{Ad}\right), 1.99$ (s, 3H, 3 $\mathrm{CH}_{\mathrm{Ad}}$ ), 6.07 (br, 2H, $\left.\mathrm{CH}_{2} \mathrm{~N}\right), 8.19-9.27$ (m, 9H, $\mathrm{CH}_{\text {arom }}$ ), 10.48 (br, 1H, NH); ${ }^{13} \mathrm{C}$ NMR (DMSO$\left.d_{6}\right): \delta 14.43\left(\mathrm{CH}_{3}\right), 27.81\left(\mathrm{CH}_{\mathrm{Ad}}\right), 34.45\left(\mathrm{CH}_{2} \mathrm{~N}\right), 36.07\left(\mathrm{CH}_{2} \mathrm{Ad}\right), 36.53\left(\mathrm{CH}_{2} \mathrm{Ad}\right), 53.90(\mathrm{CH}-\mathrm{N})$, $59.61\left(\mathrm{C}_{\mathrm{Ad}}\right), 119.45-139.00\left(\mathrm{CH}_{\text {arom }}\right.$ and $\left.\mathrm{C}_{\text {arom }}\right), 155.35\left(\mathrm{C}=\mathrm{N}^{+}\right), 163.58(\mathrm{C}=\mathrm{O})$. Anal. Calcd. for $\mathrm{C}_{27} \mathrm{H}_{31} \mathrm{BrN}_{2} \mathrm{O}$ (479.45): C, 67.64; H, 6.52; N, 5.84. Found: $\mathrm{C}, 67.34 ; \mathrm{H}, 6.32 ; \mathrm{N}, 5.60$.

5-[2-(tert-Butylamino)-2-oxoethyl]phenantridinium bromide (3c). Green-grey crystals (from toluene) (3.84 g, 70\%), mp 173-175 ${ }^{\circ} \mathrm{C}$; IR (KBr): $3208(\mathrm{NH}), 3052\left(\mathrm{CH}_{\text {arom }}\right), 2961,2865,1680$ $(\mathrm{C}=\mathrm{O}), 1626\left(\mathrm{C}=\mathrm{N}^{+}\right), 1545,1452,1363,1223,1157,754,716 \mathrm{~cm}^{-1} ;{ }^{1} \mathrm{H}$ NMR (DMSO- $\left.d_{6}\right): \delta$ $1.31\left(\mathrm{~s}, 9 \mathrm{H}, 3 \mathrm{CH}_{3}\right), 5.87\left(\mathrm{~s}, 2 \mathrm{H}, \mathrm{CH}_{2}\right), 8.09-9.22\left(\mathrm{~m}, 9 \mathrm{H}, \mathrm{CH}_{\text {arom}}\right), 10.37(\mathrm{~s}, 1 \mathrm{H}, \mathrm{NH}) ;{ }^{13} \mathrm{C}$ NMR $\left(\mathrm{DMSO}-d_{6}\right): \delta 28.38\left(\mathrm{CH}_{3}\right), 51.44(\mathrm{C}-\mathrm{N}), 119.11-138.74\left(\mathrm{CH}_{\text {arom }}\right.$ and $\left.\mathrm{C}_{\text {arom }}\right), 157.31\left(\mathrm{C}=\mathrm{N}^{+}\right)$, $163.08(\mathrm{C}=\mathrm{O})$. Anal. Calcd. for $\mathrm{C}_{19} \mathrm{H}_{21} \mathrm{BrN}_{2} \mathrm{O}$ (373.29): C, 61.13; H, 5.67; N, 7.50. Found: $\mathrm{C}$, $60.99 ; \mathrm{H}, 5.45 ; \mathrm{N}, 7.26$.

5-[2-(Ethylamino)-2-oxoethyl]phenantridinium bromide (3d). Light grey crystals (from toluene) (3.14 g, 62\%), mp 152-154 ${ }^{\circ} \mathrm{C}$; IR (KBr): $3189(\mathrm{NH}), 3048(\mathrm{C}-\mathrm{H}$ arom), 2936, 2889, $1678(\mathrm{C}=\mathrm{O}), 1625\left(\mathrm{C}=\mathrm{N}^{+}\right), 1556,1450,1354,1274,1224,1151,926,761,714 \mathrm{~cm}^{-1} ;{ }^{1} \mathrm{H} \mathrm{NMR}$ (DMSO-d $): \delta 1.10\left(\mathrm{t}, 3 \mathrm{H}, \mathrm{CH}_{3}\right), 3.19\left(\mathrm{q}, 2 \mathrm{H}, \mathrm{CH}_{2} \mathrm{~N}\right), 5.95\left(\mathrm{~s}, 2 \mathrm{H}, \mathrm{CH}_{2} \mathrm{~N}^{+}\right), 8.06-9.19(\mathrm{~m}, 9 \mathrm{H}$, $\left.\mathrm{CH}_{\text {arom}}\right), 10.46(\mathrm{~s}, 1 \mathrm{H}, \mathrm{NH}) ;{ }^{13} \mathrm{C} \mathrm{NMR}\left(\mathrm{DMSO}-d_{6}\right): \delta 14.31\left(\mathrm{CH}_{3}\right), 34.03\left(\mathrm{CH}_{2} \mathrm{~N}\right), 59.06$ $\left(\mathrm{CH}_{2} \mathrm{~N}^{+}\right), 119.15-138.52\left(\mathrm{CH}_{\text {arom }}\right.$ and $\left.\mathrm{C}_{\text {arom }}\right), 157.11\left(\mathrm{C}=\mathrm{N}^{+}\right), 163.54(\mathrm{C}=\mathrm{O})$. Anal. Calcd. for $\mathrm{C}_{17} \mathrm{H}_{17} \mathrm{BrN}_{2} \mathrm{O}$ (345.23): C, 59.14; H, 4.96; N, 8.11. Found: C, 59.00; H, 4.74; N, 7.99.

5-[2-(Benzylamino)-2-oxoethyl]phenantridinium bromide ${ }^{7}$ (3e). Green-grey crystals (from toluene) (3.41 g, 57\%), mp 165-168 ${ }^{\circ} \mathrm{C}$; IR (KBr): $3447(\mathrm{~N}-\mathrm{H}), 3000\left(\mathrm{C}-\mathrm{H}_{\text {arom }}\right), 2689,1679$ $(\mathrm{C}=\mathrm{O}), 1626\left(\mathrm{C}=\mathrm{N}^{+}\right), 1542,1455,1364,1228,1159,751,715 \mathrm{~cm}^{-1} ;{ }^{1} \mathrm{H}$ NMR (DMSO-d $): \delta$ $\left.4.38\left(\mathrm{~d}, 2 \mathrm{H}, \mathrm{CH}_{2} \mathrm{~N}\right), 6.00\left(\mathrm{~s}, 2 \mathrm{H}, \mathrm{CH}_{2} \mathrm{~N}^{+}\right), 7.25-7.34 \mathrm{CH}_{\text {arom-Bn }}\right), 8.08-9.35$ (m, 9H, $\mathrm{CH}_{\text {arom }}$ ), $10.37(\mathrm{~s}, 1 \mathrm{H}, \mathrm{NH}) ;{ }^{13} \mathrm{C}$ NMR (DMSO- $\left.d_{6}\right): \delta 42.82\left(\mathrm{CH}_{2} \mathrm{~N}\right), 59.24\left(\mathrm{CH}_{2} \mathrm{~N}^{+}\right), 119.15-138.78$ $\left(\mathrm{CH}_{\text {arom }}\right.$ and $\left.\mathrm{C}_{\text {arom }}\right), 157.20\left(\mathrm{C}=\mathrm{N}^{+}\right), 164.12(\mathrm{C}=\mathrm{O})$. Anal. Calcd. for $\mathrm{C}_{22} \mathrm{H}_{19} \mathrm{BrN}_{2} \mathrm{O}$ (407.30): $\mathrm{C}$, 64.87; H, 4.70; N, 6.88. Found: C, 64.54; H, 4.52; N, 6.68 . 
1,2,3,12b-Tetrahydropyrrolo[1,2-f]phenanthridine derivatives. General procedure. To a suspension of salt 3a-e $(2.22 \mathrm{mmol})$ in dry dichloromethane $(20 \mathrm{~mL}$; Method 1 and 2) or dry $\mathrm{CHCl}_{3}(20 \mathrm{~mL}$, Method 3) the dipolarophile $5(2.22 \mathrm{mmol})$ was added, and argon was introduced under stirring at ambient temperature. After 5 minutes dry triethylamine (224 mg, $2.22 \mathrm{mmol}$ ) was added under argon. The reaction mixture was left at ambient temperature (Method 1) or was refluxed for 2 days (Methods 2 and 3). Then the solvent was removed in vacuo, and the residue was analyzed by TLC and ${ }^{1} \mathrm{H}$ NMR. Products were separated by column chromatography on silica. Yields are listed in Table 1 (products with 5a,b) and Table 2 (products with 5c).

Products from the reaction of salts 3a-e with fumaronitrile 5a. N3-(1-Adamantyl)$\left(1 R^{*}, 2 R^{*}, 3 S^{*}, 12 \mathrm{~b} R^{*}\right)-1,2$-dicyano-1,2,3,12b-tetrahydropyrrolo[1,2-f]phenanthridine-3carboxamide $^{7}$ (6aa)

Yellowish crystals (from chloroform/ether), $\mathrm{mp} \quad 133-135{ }^{\circ} \mathrm{C} ; \quad \mathrm{R}_{f}=0.85$ (silica; dichloromethane/ether 4:1); IR (KBr): $3342(\mathrm{~N}-\mathrm{H}), 3061(\mathrm{C}-\mathrm{H}$ arom), 2908, 2851, 2364, $(\mathrm{C} \equiv \mathrm{N})$, $2344(\mathrm{C} \equiv \mathrm{N}), 1678(\mathrm{C}=\mathrm{O}), 1602,1519,1444,1263,1094,755 \mathrm{~cm}^{-1} ;{ }^{1} \mathrm{H} \mathrm{NMR}\left(\mathrm{CDCl}_{3}\right): \delta 1.66-$ $1.97\left(\mathrm{~m}, 12 \mathrm{H}, 6 \mathrm{CH}_{2 \mathrm{Ad}}\right), 2.07\left(\mathrm{~s}, 3 \mathrm{H}, 3 \mathrm{CH}_{\mathrm{Ad}}\right), 3.19(\mathrm{dd}, J=9.7 \mathrm{~Hz}, J=9.7 \mathrm{~Hz}, 1 \mathrm{H}, \mathrm{H}-2), 3.93$ $(\mathrm{dd}, J=9.5 \mathrm{~Hz}, J=4.7 \mathrm{~Hz}, 1 \mathrm{H}, \mathrm{H}-1), 4.62$ (d, $J=10.0 \mathrm{~Hz}, 1 \mathrm{H}, \mathrm{H}-3), 4.85$ (d, $J=5.2 \mathrm{~Hz}, 1 \mathrm{H}, \mathrm{H}-$ 12b), 6.39 (s, 1H, NH), 6.79-7,87 (m, 8H, C-H arom); ${ }^{13} \mathrm{C} \mathrm{NMR}\left(\mathrm{CDCl}_{3}\right): \delta 29.25\left(\mathrm{CH}_{\mathrm{Ad}}\right), 33.54$ $(\mathrm{C}-2), 36.01\left(\mathrm{CH}_{2} \mathrm{Ad}\right), 41.05(\mathrm{C}-1), 41.33\left(\mathrm{CH}_{2} \mathrm{Ad}\right), 52.56\left(\mathrm{C}_{\mathrm{Ad}}\right), 64.20(\mathrm{C}-3), 69.53(\mathrm{C}-12 \mathrm{~b})$, 113.31-140.74 $\left(\mathrm{CH}_{\text {arom }}\right.$ and $\left.\mathrm{C}_{\text {arom }}\right), 116.28(\mathrm{C} \equiv \mathrm{N}), 117.87(\mathrm{C} \equiv \mathrm{N}), 166.70(\mathrm{C}=\mathrm{O}) ; \mathrm{EI}-\mathrm{MS}, \mathrm{m} / z$ (\%): $448\left(\mathrm{M}^{+}, 1\right), 421$ (2), 419 (3), 370 (1), 313 (1), 270 (12), 219 (22), 218 (96), 180 (12), 135 (16). Anal. Calcd. for $\mathrm{C}_{29} \mathrm{H}_{28} \mathrm{~N}_{4} \mathrm{O}$ (448.56): C, 77.65; H, 6.29; N, 12.49. Found: C, 77.37; H, $6.11 ; \mathrm{N}, 12.25$.

\section{$N 3-(1-A d a m a n t y l)-\left(1 R^{*}, 2 R^{*}, 3 R^{*}, 12 \mathrm{~b} S^{*}\right)-1,2$-dicyano-1,2,3,12b-tetrahydropyrrolo[1,2-}

flphenanthridine-3-carboxamide ${ }^{7}$ (7aa). Dark yellow crystals (from chloroform/ether), mp 157-159 ${ }^{\circ} \mathrm{C} ; \mathrm{R}_{f}=0.68$ (silica; dichloromethane/ether 4:1); IR (KBr): $3361(\mathrm{NH}), 3064(\mathrm{C}-\mathrm{H}$ arom), 2907, 2851, $2354(\mathrm{C} \equiv \mathrm{N}), 2339(\mathrm{C} \equiv \mathrm{N}), 1674(\mathrm{C}=\mathrm{O}), 1603,1522,1444,1263,1094,746$ $\mathrm{cm}^{-1} ;{ }^{1} \mathrm{H}$ NMR $\left(\mathrm{CDCl}_{3}\right): \delta$ 1.68-2.06 (m, $\left.12 \mathrm{H}, 6 \mathrm{CH}_{2} \mathrm{Ad}\right), 2.09\left(\mathrm{~s}, 3 \mathrm{H}, 3 \mathrm{CH}_{\mathrm{Ad}}\right), 3.67(\mathrm{~d}, J=4.1$ Hz, 1H, H-2), 3.99 (d, J=8.3 Hz, 1H, H-1), 4.57 (d, $J=8.3 \mathrm{~Hz}, 1 \mathrm{H}, \mathrm{H}-12 \mathrm{~b}), 5.54$ (d, $J=4.2 \mathrm{~Hz}$, $1 \mathrm{H}, \mathrm{H}-3), 6.48$ (s, $1 \mathrm{H}, \mathrm{NH}),(6.93-7.92)\left(\mathrm{m}, 8 \mathrm{H}, \mathrm{CH}_{\text {arom }}\right) ;{ }^{13} \mathrm{C} \mathrm{NMR}\left(\mathrm{CDCl}_{3}\right): \delta 29.28\left(\mathrm{CH}_{\mathrm{Ad}}\right)$, $35.65(\mathrm{C}-2), 36.06\left(\mathrm{CH}_{2} \mathrm{Ad}\right), 41.26\left(\mathrm{CH}_{2} \mathrm{Ad}\right), 42.96(\mathrm{C}-1), 52.59\left(\mathrm{C}_{\mathrm{Ad}}\right), 62.94(\mathrm{C}-12 \mathrm{~b}), 68.81(\mathrm{C}-$ 3), 113.01-142.09 ( $\mathrm{CH}_{\text {arom }}$ and $\left.\mathrm{C}_{\text {arom }}\right), 115.00(\mathrm{C} \equiv \mathrm{N}), 115.98(\mathrm{C} \equiv \mathrm{N}), 166.08(\mathrm{C}=\mathrm{O})$; EI-MS, $m / z$ (\%): $448\left(\mathrm{M}^{+}, 1\right), 421$ (2), 419 (3), 370 (1), 313 (1), 270 (12), 219 (22), 218 (96), 180 (12), 135 (16). Anal. Calcd. for $\mathrm{C}_{29} \mathrm{H}_{28} \mathrm{~N}_{4} \mathrm{O}$ (448.56): C, 77.65; H, 6.29; N, 12.49. Found: C, 77.45; H, $6.12 ; \mathrm{N}, 12.22$.

N3-(1-Adamantyl)-1,2-dicyanopyrrolo[1,2-f]phenanthridine-3-carboxamide (9aa). White crystals (insoluble in most solvents), $\mathrm{mp}>350^{\circ} \mathrm{C} ; \mathrm{R}_{f}=0.73$ (silica; dichloromethane/ether 25:4); 
IR (KBr): $3344(\mathrm{NH}), 3069\left(\mathrm{CH}_{\text {arom }}\right)$, 2910, 2852, $2217(\mathrm{C} \equiv \mathrm{N}), 1672(\mathrm{C}=\mathrm{O})$, 1525, 1444, 1364, 1296, 1092, $754 \mathrm{~cm}^{-1}$; EI-MS, m/z (\%): $444\left(\mathrm{M}^{+}, 100\right), 420$ (2), 419 (2), 387 (41), 309 (21), 294 (65), 281(7), 267 (19), 266 (26), 265 (27), 239 (7), 216 (16), 199 (4), 135 (54). Anal. Calcd. for $\mathrm{C}_{29} \mathrm{H}_{24} \mathrm{~N}_{4} \mathrm{O}$ (444.53): C, 78.36; H, 5.44; N, 12.60; Found: C, 78.13, H, 5.36; N, 12.45.

N3-[(1RS)-1-(1-Adamantyl)-1-ethyl]-(1R*,2R*,3S*,12b $\left.R^{*}\right)-1,2$-dicyano-1,2,3,12btetrahydropyrrolo[1,2-f]phenanthridine-3-carboxamide ${ }^{7}$ (6ba). Yellowish crystals (from chloroform/ ether), mp 195-197 ${ }^{\circ} \mathrm{C} ; \mathrm{R}_{f}=0.74$ (silica; ethyl acetate); IR (KBr): $3299(\mathrm{NH}), 3061$ (C-Harom), 2903, 2847, $2247(\mathrm{C} \equiv \mathrm{N}), 1674(\mathrm{C}=\mathrm{O}), 1603,1522,1495,11447,1383,750 \mathrm{~cm}^{-1} ;{ }^{1} \mathrm{H}$ $\operatorname{NMR}\left(\mathrm{CDCl}_{3}\right): \delta 1.04\left(\mathrm{~d}, J=6.9 \mathrm{~Hz}, 3 \mathrm{H}, \mathrm{CH}_{3}\right), 1.36(\mathrm{q}, J=6.9 \mathrm{~Hz}, 1 \mathrm{H}, \mathrm{HC}-\mathrm{N}), 1.53-1.62(\mathrm{~m}$, $12 \mathrm{H}, 6 \mathrm{CH}_{2 \mathrm{Ad}}$ ), 1.99 (s, 3H, $3 \mathrm{CH}_{\mathrm{Ad}}$ ), 3.23 (dd, $\left.J=9.9, J=9.9, \mathrm{~Hz}, 1 \mathrm{H}, \mathrm{H}-2\right), 3.27$ (dd, $J=10.0$ $\mathrm{Hz}, J=10.0 \mathrm{~Hz}, 1 \mathrm{H}, \mathrm{H}-2), 3.94(\mathrm{dd}, J=9.9 \mathrm{~Hz}, J=4.6 \mathrm{~Hz}, 1 \mathrm{H}, \mathrm{H}-1), 4.01(\mathrm{dd}, J=10.0 \mathrm{~Hz}, J=$ $4.5 \mathrm{~Hz}, 1 \mathrm{H}, \mathrm{H}-1), 4.70$ (d, $J=9.9 \mathrm{~Hz}, 1 \mathrm{H}, \mathrm{H}-3), 4.72$ (d, $J=10.0 \mathrm{~Hz}, 1 \mathrm{H}, \mathrm{H}-3), 4.95$ (d, $J=4.6$ $\mathrm{Hz}, 1 \mathrm{H}, \mathrm{H}-12 \mathrm{~b}), 5.00$ (d, J=4.5 Hz, 1H, H-12b), 6.48 (br, 1H, N-H), 6.82-7.90 (m, 8H, CH $\mathrm{Crom}_{\text {); }}$ ${ }^{13} \mathrm{C} \mathrm{NMR}\left(\mathrm{CDCl}_{3}\right): \delta 14.45\left(\mathrm{CH}_{3}\right), 28.13\left(\mathrm{CH}_{\mathrm{Ad}}\right), 33.89(\mathrm{C}-2), 34.06(\mathrm{C}-2), 36.10\left(\mathrm{C}_{\mathrm{Ad}}\right), 36.88$ $\left(\mathrm{CH}_{2 \mathrm{Ad}}\right), 38.60\left(\mathrm{CH}_{2 \mathrm{Ad}}\right), 40.84(\mathrm{C}-1), 41.22(\mathrm{C}-1), 53.69(\mathrm{C}-3), 53.78(\mathrm{C}-3), 64.05(\mathrm{CH}-\mathrm{N})$, $64.80(\mathrm{CH}-\mathrm{N}), 69.03(\mathrm{C}-12 \mathrm{~b}), 69.06(\mathrm{C}-12 \mathrm{~b}), 113.21140 .82\left(\mathrm{CH}_{\text {aro }}\right.$ and $\left.\mathrm{C}_{\text {arom}}\right), 116.23,116.31$, $117.76(\mathrm{C} \equiv \mathrm{N}), 117.94(\mathrm{C} \equiv \mathrm{N}), 167.17(\mathrm{C}=\mathrm{O})$; EI-MS: $m / z(\%) 476\left(\mathrm{M}^{+}, 3\right), 449(1), 423(1), 313$ (2), 270 (19), 243 (11), 219 (20), 218 (100), 193 (8), 180 (25), 179 (18), 165 (7), 135 (11), 79 (18), 67 (7), 41 (6). Anal. Calcd. for $\mathrm{C}_{31} \mathrm{H}_{32} \mathrm{~N}_{4} \mathrm{O}$ (476.62): C, 78.12; H, 6.77; N, 11.75. Found: C, 77.98; H, 6.55; N, 11.55 .

N3-(tert-Butyl)-(1 $\left.R^{*}, 2 R^{*}, 3 S^{*}, 12 \mathrm{~b} R^{*}\right)-1,2$-dicyano-1,2,3,12b-tetrahydropyrrolo[1,2-

flphenanthridine-3-carboxamide (6ca). Yellowish crystals (from chloroform/ether), mp 136$138{ }^{\circ} \mathrm{C} ; \mathrm{R}_{f}=0.61$ (silica; dichloromethane/ethyl acetate 5:1); IR (KBr): $3350(\mathrm{~N}-\mathrm{H}), 3069(\mathrm{C}-$ Harom), 2968, 2932, $2249(\mathrm{C} \equiv \mathrm{N}), 2184(\mathrm{C} \equiv \mathrm{N}), 1676(\mathrm{C}=\mathrm{O}), 1632,1523,1448,1260,1097,753$ $\mathrm{cm}^{-1} ;{ }^{1} \mathrm{H}$ NMR $\left(\mathrm{CDCl}_{3}\right): \delta 1.39\left(\mathrm{~s}, 9 \mathrm{H}, 3 \mathrm{CH}_{3}\right), 3.23(\mathrm{dd}, J=9.9 \mathrm{~Hz}, J=9.9 \mathrm{~Hz}, 1 \mathrm{H}, \mathrm{H}-2), 3.98$ $(\mathrm{dd}, J=9.9 \mathrm{~Hz}, J=4.7 \mathrm{~Hz}, 1 \mathrm{H}, \mathrm{H}-1), 4.64$ (d, $J=9.9 \mathrm{~Hz}, 1 \mathrm{H}, \mathrm{H}-3), 4.90$ (d, $J=4.7 \mathrm{~Hz}, 1 \mathrm{H}, \mathrm{H}-$ 12b), 6.57 (s, 1H, NH), 6.82-7.91 (m, 8H, $\left.\mathrm{CH}_{\text {arom }}\right) ;{ }^{13} \mathrm{C} \mathrm{NMR}\left(\mathrm{CDCl}_{3}\right): \delta 27.64\left(\mathrm{CH}_{3}\right), 32.61(\mathrm{C}-$ 2), $40.15(\mathrm{C}-1), 51.02(\mathrm{C}-\mathrm{N}), 63.37(\mathrm{C}-3), 68.69(\mathrm{C}-12 \mathrm{~b}), 112.39-139.83\left(\mathrm{CH}_{\text {arom }}\right.$ and $\left.\mathrm{C}_{\text {arom }}\right)$, $115.35(\mathrm{C} \equiv \mathrm{N}), 116.94(\mathrm{C} \equiv \mathrm{N}), 166.15(\mathrm{C}=\mathrm{O})$; EI-MS: m/z (\%) $370\left(\mathrm{M}^{+}, 1\right), 343(1), 313(1), 277$ (1), 271 (3), 270 (17), 268 (7), 243 (6), 219 (17), 218 (100), 193 (33), 179 (22), 58 (5), 41 (4). Anal. Calcd. for $\mathrm{C}_{23} \mathrm{H}_{22} \mathrm{~N}_{4} \mathrm{O}$ (370.45): C, 74.57; H, 5.99; N, 15.12. Found: C, 74.33; H, 5.61; N, 15.22 .

\section{N3-(tert-Butyl)-(1 $\left.R^{*}, 2 R^{*}, 3 R^{*}, 12 \mathrm{bS} *\right)-1,2$-dicyano-1,2,3,12b-tetrahydropyrrolo[1,2-}

flphenanthridine-3-carboxamide (7ca). Light yellow crystals (from chloroform/ether), mp 156-157 ${ }^{\circ} \mathrm{C} ; \mathrm{R}_{f}=0.32$ (silica; dichloromethane/ethyl acetate 5:1); IR (KBr): $3365(\mathrm{~N}-\mathrm{H}), 3070$ $\left(\mathrm{CH}_{\text {arom }}\right)$, 2971, 2941, $2252(\mathrm{C} \equiv \mathrm{N}), 2189(\mathrm{C} \equiv \mathrm{N}), 1678(\mathrm{C}=\mathrm{O}), 1638,1523,1449,1261,1099$, $756 \mathrm{~cm}^{-1} ;{ }^{1} \mathrm{H}$ NMR $\left(\mathrm{CDCl}_{3}\right): \delta 1.41\left(\mathrm{~s}, 9 \mathrm{H}, 3 \mathrm{CH}_{3}\right), 3.68(\mathrm{~d}, J=3.5 \mathrm{~Hz}, 1 \mathrm{H}, \mathrm{H}-2), 4.01(\mathrm{~d}, J=$ 8.7 Hz, 1H, H-1), 4.59 (d, J=8.7 Hz, 1H, H-12b), 5.55 (d, J=3.5 Hz, 1H, H-3), 6.57 (s, 1H, N- 
$\mathrm{H}), 6.82-7.91\left(\mathrm{~m}, 8 \mathrm{H}, \mathrm{CH}_{\text {arom }}\right) ;{ }^{13} \mathrm{C} \mathrm{NMR}\left(\mathrm{CDCl}_{3}\right): \delta 27.63\left(\mathrm{CH}_{3}\right), 34.68(\mathrm{C}-2), 42.06(\mathrm{C}-1)$, $51.07\left[\underline{C}\left(\mathrm{CH}_{3}\right)_{3}\right], 62.05(\mathrm{C}-12 \mathrm{~b}), 67.88(\mathrm{C}-3), 112.03-144.52\left(\mathrm{CH}_{\text {arom }}\right.$ and $\left.\mathrm{C}_{\text {arom }}\right), 114.12(\mathrm{C} \equiv \mathrm{N})$, $115.07(\mathrm{C} \equiv \mathrm{N}), 166.15(\mathrm{C}=\mathrm{O})$; EI-MS: m/z (\%) $370\left(\mathrm{M}^{+}, 1\right), 343$ (1), 313 (1), 277 (1), 271 (3), 270 (17), 268 (7), 243 (6), 219 (17), 218 (100), 193 (33), 179 (22), 58 (5), 41 (4). Anal. Calcd. for $\mathrm{C}_{23} \mathrm{H}_{22} \mathrm{~N}_{4} \mathrm{O}$ (370.45): C, 74.57; H, 5.99; N, 15.12. Found: C, 74.40; H, 5.88; N, 15.01 .

N3-(tert-Butyl)-(2R*,3R*)-1,2-dicyano-2,3-dihydropyrrolo[1,2-f]phenanthridine-3-

carboxamide (8ca). Yellow crystals (from chloroform/ether), mp $279-281^{\circ} \mathrm{C} ; \mathrm{R}_{f}=0.68$ (silica; petroleum ether/ethyl acetate 5:1); IR (KBr): $3315(\mathrm{~N}-\mathrm{H}), 3067\left(\mathrm{CH}_{\text {arom }}\right), 2906,2847,2249$ $(\mathrm{C} \equiv \mathrm{N}), 2186(\mathrm{C} \equiv \mathrm{N}), 1657(\mathrm{C}=\mathrm{O}), 1602,1547,1448,1383,1094,748 \mathrm{~cm}^{-1} ;{ }^{1} \mathrm{H} \mathrm{NMR}\left(\mathrm{CDCl}_{3}\right): \delta$ 1.39 (s, 9H, $3 \mathrm{CH}_{3}$ ), 5.23 (d, $\left.J=4.9 \mathrm{~Hz}, 1 \mathrm{H}, \mathrm{H}-2\right), 5.97$ (d, $\left.J=4.9 \mathrm{~Hz}, 1 \mathrm{H}, \mathrm{H}-3\right), 6.59$ (s, 1H, $\mathrm{NH}), 6.82-7.91\left(\mathrm{~m}, 8 \mathrm{H}, \mathrm{CH}_{\text {arom }}\right) ;{ }^{13} \mathrm{C} \mathrm{NMR}\left(\mathrm{CDCl}_{3}\right): \delta 27.64\left(\mathrm{CH}_{3}\right), 51.02\left[\underline{C}\left(\mathrm{CH}_{3}\right)_{3}\right], 59.61(\mathrm{C}-$ 2), 65.28 (C-3), $93.54(\mathrm{C}-1), 112.39-139.83\left(\mathrm{CH}_{\text {arom }}\right.$ and $\left.\mathrm{C}_{\text {arom }}\right), 115.35(\mathrm{C} \equiv \mathrm{N}), 116.94(\mathrm{C} \equiv \mathrm{N})$, $166.15(\mathrm{C}=\mathrm{O})$; EI-MS: m/z (\%) $368\left(\mathrm{M}^{+}, 15\right), 343$ (12), 313 (5), 270 (77), 241 (7), 216 (100), 193 (33), 178 (22), 58 (5), 41 (4). Anal. Calcd. for $\mathrm{C}_{23} \mathrm{H}_{20} \mathrm{~N}_{4} \mathrm{O}$ (368.43): C, 74.98; H, 5.47; N, 15.21. Found: C, 74.82; H, 5.15; N, 15.03 .

N3-(tert-Butyl)-1,2-dicyanopyrrolo[1,2-f]phenanthridine-3-carboxamide (9ca). Pale pink crystals (insoluble in common solvents), $\mathrm{mp}>350{ }^{\circ} \mathrm{C} ; \mathrm{R}_{f}=0.62$ (silica; dichloromethane/ether 15:1); IR (KBr): $3358(\mathrm{NH}), 3069\left(\mathrm{C}-\mathrm{H}_{\text {arom }}\right), 2968,2927,2220(\mathrm{C} \equiv \mathrm{N}), 1670(\mathrm{C}=\mathrm{O}), 1601,1566$, 1447, 1365, 1217, 1085, $750 \mathrm{~cm}^{-1}$; EI-MS: m/z (\%) $366\left(\mathrm{M}^{+}, 100\right), 310$ (19), 309 (90), 294 (95), 267 (35), 242 (19), 239 (18), 218 (11), 57 (18), 41 (12). Anal. Calcd. for $\mathrm{C}_{23} \mathrm{H}_{18} \mathrm{~N}_{4} \mathrm{O}$ (366.42): C, 75.39; H, 4.95; N; 15.29. Found: C, 75.32; H, 5.06; N, 15.32.

$N 3$-Ethyl-(1R*,2R*,3S*,12bR*)-1,2-dicyano-1,2,3,12b-tetrahydropyrrolo[1,2-

flphenanthridine-3-carboxamide (6da). Yellowish crystals (from chloroform/ether), mp 227$228^{\circ} \mathrm{C} ; \mathrm{R}_{f}=0.72$ (silica; ethyl acetate); IR (KBr): $3390(\mathrm{~N}-\mathrm{H}), 3065\left(\mathrm{CH}_{\text {arom }}\right), 2976,2934,2239$ $(\mathrm{C} \equiv \mathrm{N}), 2185(\mathrm{C} \equiv \mathrm{N}), 1669(\mathrm{C}=\mathrm{O}), 1601,1532,1445,1261,961,752 \mathrm{~cm}^{-1} ;{ }^{1} \mathrm{H} \mathrm{NMR}\left(\mathrm{CDCl}_{3}\right): \delta$ $1.18\left(\mathrm{t}, 3 \mathrm{H}, \mathrm{CH}_{3}\right), 3.24(\mathrm{dd}, J=9.9 \mathrm{~Hz}, J=9.9 \mathrm{~Hz}, 1 \mathrm{H}, \mathrm{H}-2), 3.45$ (q, 2H, $\left.\mathrm{CH}_{2}\right), 3.97$ (dd, $J=9.9$ $\mathrm{Hz}, J=4.7 \mathrm{~Hz}, 1 \mathrm{H}, \mathrm{H}-1), 4.70$ (d, $J=9.9 \mathrm{~Hz}, 1 \mathrm{H}, \mathrm{H}-3), 4.98$ (d, $J=4.7 \mathrm{~Hz}, 1 \mathrm{H}, \mathrm{H}-12 \mathrm{~b}), 6.48$ (s, $1 \mathrm{H}, \mathrm{N}-\mathrm{H}), 6.82-7.94\left(\mathrm{~m}, 8 \mathrm{H}, \mathrm{CH}_{\text {arom }}\right) ;{ }^{13} \mathrm{C} \mathrm{NMR}\left(\mathrm{CDCl}_{3}\right): \delta 14.61\left(\mathrm{CH}_{3}\right), 33.76(\mathrm{C}-2), 34.89$ $\left(\mathrm{CH}_{2} \mathrm{~N}\right), 41.06(\mathrm{C}-1), 64.28(\mathrm{C}-3), 69.00(\mathrm{C}-12 \mathrm{~b}), 116.28-141.98\left(\mathrm{CH}_{\text {arom }}\right.$ and $\left.\mathrm{C}_{\text {arom}}\right), 116.28$ $(\mathrm{C} \equiv \mathrm{N}), 117.92(\mathrm{C} \equiv \mathrm{N}), 167.99(\mathrm{C}=\mathrm{O})$; EI-MS: $m / z(\%) 341\left(\mathrm{M}^{+}, 1\right), 294$ (1), 270 (12), 219 (18), 218 (100), 204 (3), 193 (6), 180 (10), 179 (9), 165 (8), 135 (8), 106 (3), 93 (8), 79 (10), 67 (5), 55 (3), 41 (3). Anal. Calcd. for $\mathrm{C}_{21} \mathrm{H}_{18} \mathrm{~N}_{4} \mathrm{O}$ (342.40): C, 73.67; H, 5.30; N, 16.36. Found: C, $73.55 ; \mathrm{H}, 5.11 ; \mathrm{N}, 16.09$.

\section{$N 3$-Benzyl-(1R*,2R*,3S*,12bR*)-1,2-dicyano-1,2,3,12b-tetrahydropyrrolo[1,2-}

flphenanthridine-3-carboxamide (6ea). Dark yellow crystals (from chloroform/ether), mp 238-239 ${ }^{\circ} \mathrm{C} ; \mathrm{R}_{f}=0.71$ (silica; ethyl acetate/petroleum ether 2.5:1); IR (KBr): $3325(\mathrm{~N}-\mathrm{H}), 3066$ $\left(\mathrm{CH}_{\text {arom }}\right)$, 3032, 2889, $2178(\mathrm{C} \equiv \mathrm{N}), 2177(\mathrm{C} \equiv \mathrm{N}), 1652(\mathrm{C}=\mathrm{O}), 1603,1563,1449,1365,1233$, $1175,749 \mathrm{~cm}^{-1} ;{ }^{1} \mathrm{H}$ NMR $\left(\mathrm{CDCl}_{3}\right): \delta 4.29(\mathrm{dd}, J=9.9 \mathrm{~Hz}, J=9.9 \mathrm{~Hz}, 1 \mathrm{H}, \mathrm{H}-2), 4.37(\mathrm{dd}, J=$ 
$9.9 \mathrm{~Hz}, J=4.0 \mathrm{~Hz}, 1 \mathrm{H}, \mathrm{H}-1), 4.43\left(\mathrm{~d}, 2 \mathrm{H}, \mathrm{CH}_{2} \mathrm{~N}\right), 4.78$ (d, J=9.9 Hz, 1H, H-3), 5.29 (d, $J=4.0$ $\mathrm{Hz}, 1 \mathrm{H}, \mathrm{H}-12 \mathrm{~b}), 6.48(\mathrm{~s}, 1 \mathrm{H}, \mathrm{N}-\mathrm{H}), 6.81-8.98\left(\mathrm{~m}, 8 \mathrm{H}, \mathrm{CH}_{\text {arom}}\right) ;{ }^{13} \mathrm{C} \mathrm{NMR}\left(\mathrm{CDCl}_{3}\right): \delta 37.60(\mathrm{C}-$ 2), $38.85(\mathrm{C}-1), 43.61\left(\mathrm{CH}_{2} \mathrm{~N}\right), 66.13(\mathrm{C}-3), 68.10(\mathrm{C}-12 \mathrm{~b}), 113.36-144.12\left(\mathrm{CH}_{\text {arom }}\right.$ and $\left.\mathrm{C}_{\text {arom }}\right)$, $117.28(\mathrm{C} \equiv \mathrm{N}), 117.63(\mathrm{C} \equiv \mathrm{N}), 166.50(\mathrm{C}=\mathrm{O})$; EI-MS: $m / z(\%) 404\left(\mathrm{M}^{+}, 3\right), 294(30), 267(20)$, 218 (100), 193 (12), 180 (14). Anal. Calcd. for $\mathrm{C}_{26} \mathrm{H}_{20} \mathrm{~N}_{4} \mathrm{O}$ (404.47): C, 77.21; H, 4.98; N, 13.85. Found: C, 77.02; H, 4.69; N, 13.77.

\section{Products from the reaction of salts $3 a-d$ with dimethyl fumarate $5 b$}

Dimethyl $\left(1 R^{*}, 2 R^{*}, 3 S *, 12 b R^{*}\right)-3-(1-$ adamantyl $)$ aminocarbonyl-1, $2,3,12 b-$ tetrahydropyrrolo-[1,2-f]phenanthridine-1,2-dicarboxylate ${ }^{7}$ (6ab). Dark orange crystals (from chloroform/ether), mp 191-193 ${ }^{\circ} \mathrm{C} ; \mathrm{R}_{f}=0.83$ (silica; dichloromethane/ether 5:1); IR $(\mathrm{KBr}): 3300(\mathrm{~N}-\mathrm{H}), 3030\left(\mathrm{CH}_{\text {arom }}\right), 2908,2849,1730,(\mathrm{C}=\mathrm{O}), 1675(\mathrm{C}=\mathrm{O}), 1149,1167,741 \mathrm{~cm}^{-}$ 1. ${ }^{1} \mathrm{H} \mathrm{NMR}\left(\mathrm{CDCl}_{3}\right): \delta 1.67-1.97\left(\mathrm{~m}, 12 \mathrm{H}, 6 \mathrm{CH}_{2 \mathrm{Ad}}\right), 2.07\left(\mathrm{~s}, 3 \mathrm{H}, 3 \mathrm{CH}_{\mathrm{Ad}}\right), 3.38(\mathrm{dd}, J=9.5 \mathrm{~Hz}$, $J=9.5 \mathrm{~Hz}, 1 \mathrm{H}, \mathrm{H}-2), 3.73\left(\mathrm{~s}, 3 \mathrm{H}, \mathrm{OCH}_{3}\right), 3.75$ (dd, $\left.J=9.4 \mathrm{~Hz}, J=5.1 \mathrm{~Hz}, 1 \mathrm{H}, \mathrm{H}-1\right), 3.80$ (s, 3H, $\left.\mathrm{OCH}_{3}\right), 4.79$ (d, $\left.J=5.2 \mathrm{~Hz}, 1 \mathrm{H}, \mathrm{H}-12 \mathrm{~b}\right), 4.97$ (d, $\left.J=9.5 \mathrm{~Hz}, 1 \mathrm{H}, \mathrm{H}-3\right), 6.44$ (s, 1H, N-H), 6.69$8.65\left(\mathrm{~m}, 8 \mathrm{H}, \mathrm{CH}_{\text {arom }}\right) ;{ }^{13} \mathrm{C} \mathrm{NMR}\left(\mathrm{CDCl}_{3}\right): \delta 29.32\left(\mathrm{CH}_{\mathrm{Ad}}\right), 36.17\left(\mathrm{CH}_{2 \mathrm{Ad}}\right), 41.41\left(\mathrm{CH}_{2} \mathrm{Ad}\right), 50.37$ $(\mathrm{C}-2), 51.87\left(\mathrm{C}_{\mathrm{Ad}}\right), 54.2\left(\mathrm{OCH}_{3}\right), 55.18(\mathrm{C}-1), 55.7\left(\mathrm{OCH}_{3}\right), 64.02(\mathrm{C}-3), 68.53(\mathrm{C}-12 \mathrm{~b}), 113.5-$ $142.97\left(\mathrm{CH}_{\text {arom }}\right.$ and $\left.\mathrm{C}_{\text {arom }}\right), 169.53,171.81,172.05$ (3 C=O); EI-MS: $m / z(\%) 514\left(\mathrm{M}^{+}, 1\right), 398$ (5), 374 (5), 304 (5), 251 (40), 219 (12), 194 (31), 180 (100), 135 (22). Anal. Calcd. for $\mathrm{C}_{31} \mathrm{H}_{34} \mathrm{~N}_{2} \mathrm{O}_{5}$ (514.62): C, 72.35; H, 6.66; N, 5.44. Found: C, 72.22; H, 6.46; N, 5.15.

Dimethyl $\quad\left(1 R^{*}, 2 R^{*}, 3 R^{*}, 12 \mathrm{~b} S *\right)-3-(1-\operatorname{adamantyl})$ aminocarbonyl-1,2,3,12 btetrahydropyrrolo-[1,2-f]phenanthridine-1,2-dicarboxylate ${ }^{7}$ (7ab). Yellowish crystals (from chloro-form/ether), mp 208-210 ${ }^{\circ} \mathrm{C} ; \mathrm{R}_{f}=0.72$ (silica; dichloromethane/ether 5:1); IR (KBr): 3399 (N-H), 3035 (C-Harom), 2909, 2855, $1729(\mathrm{C}=\mathrm{O}), 1647(\mathrm{C}=\mathrm{O}), 1450,1168,741 \mathrm{~cm}^{-1}$; ${ }^{1} \mathrm{H}$ $\operatorname{NMR}\left(\mathrm{CDCl}_{3}\right): \delta 1.68-1.97\left(\mathrm{~m}, 12 \mathrm{H}, 6 \mathrm{CH}_{2} \mathrm{Ad}\right), 2.08\left(\mathrm{~s}, 3 \mathrm{H}, 3 \mathrm{CH}_{\mathrm{Ad}}\right), 3.38(\mathrm{~d}, J=5.6 \mathrm{~Hz}, 1 \mathrm{H}, \mathrm{H}-$ 2), $3.67\left(\mathrm{~s}, 3 \mathrm{H}, \mathrm{OCH}_{3}\right), 3.80\left(\mathrm{~s}, 3 \mathrm{H}, \mathrm{OCH}_{3}\right), 3.75$ (d, $\left.J=9.2 \mathrm{~Hz}, 1 \mathrm{H}, \mathrm{H}-1\right), 4.72$ (d, J=9.1 Hz, $1 \mathrm{H}, \mathrm{H}-12 \mathrm{~b}), 5.70$ (d, $J=5.7 \mathrm{~Hz}, 1 \mathrm{H}, \mathrm{H}-3), 6.54(\mathrm{~s}, 1 \mathrm{H}, \mathrm{N}-\mathrm{H}), 6.81-8.64\left(\mathrm{~m}, 8 \mathrm{H}, \mathrm{CH}_{\text {arom}}\right) ;{ }^{13} \mathrm{C}$ NMR $\left(\mathrm{CDCl}_{3}\right): \delta 29.45\left(\mathrm{CH}_{\mathrm{Ad}}\right), 36.32\left(\mathrm{CH}_{2} \mathrm{Ad}\right), 41.38\left(\mathrm{CH}_{2 \mathrm{Ad}}\right), 46.17(\mathrm{C}-2), 51.0\left(\mathrm{OCH}_{3}\right), 53.7$ $\left(\mathrm{OCH}_{3}\right), 55.79(\mathrm{C}-1), 52.39\left(\mathrm{C}_{\mathrm{Ad}}\right), 63.30(\mathrm{C}-12 \mathrm{~b}), 71.36(\mathrm{C}-3), 112.75-144.42\left(\mathrm{CH}_{\text {arom }}\right.$ and $\mathrm{C}_{\text {arom}}$ ), 166.08, 172.49, 172.90 (3 C=O); EI-MS: m/z (\%) $514\left(\mathrm{M}^{+}, 1\right), 398$ (5), 374 (5), 304 (5), 251 (40), 219 (12), 194 (31), 180 (100), 135 (22). Anal. Calcd. for $\mathrm{C}_{31} \mathrm{H}_{34} \mathrm{~N}_{2} \mathrm{O}_{5}$ (514.62): C, 72.35; H, 6.66; N, 5.44. Found: C, 71.99; H, 6.24; N, 5.14.

Dimethyl $\left(1 R^{*}, 2 R^{*}, 3 S^{*}, 12 \mathrm{~b} R^{*}\right)-3-[(1 R S)-1-(1-a d a m a n t y l)-1-e t h y l a m i n o c a r b o n y l]-1,2,3,12 \mathrm{~b}-$ tetrahydropyrrolo[1,2-f]phenanthridine-1,2-dicarboxylate (6bb). White-yellow crystals (from chloroform/ether), mp $250-252{ }^{\circ} \mathrm{C} ; \mathrm{R}_{f}=0.66$ (silica; ethyl acetate); IR (KBr): $3418(\mathrm{NH})$, 3075, 2903, 2847, $1736(\mathrm{C}=\mathrm{O}), 1673(\mathrm{C}=\mathrm{O}), 1516,1447,1314,1163,1011,750 \mathrm{~cm}^{-1}$; ${ }^{1} \mathrm{H}$ NMR $\left(\mathrm{CDCl}_{3}\right): \delta 1.04(\mathrm{~d}, J=6.9 \mathrm{~Hz}, 3 \mathrm{H}, \mathrm{CH} 3)^{2}, 1.36$ (q, $\left.J=6.9,1 \mathrm{H}, \mathrm{HC}-\mathrm{N}\right), 1.53-1.62(\mathrm{~m}, 12 \mathrm{H}, 6$ $\left.\mathrm{CH}_{2 \mathrm{Ad}}\right), 1.99$ (s, 3H, $\left.3 \mathrm{CH}\right), 3.23(\mathrm{dd}, J=9.4 \mathrm{~Hz}, J=9.4 \mathrm{~Hz}, 1 \mathrm{H}, \mathrm{H}-2), 3.75$ (dd, $J=9.4 \mathrm{~Hz}, J=$ 
$5.0 \mathrm{~Hz}, 1 \mathrm{H}, \mathrm{H}-1), 3.82\left(\mathrm{~s}, 3 \mathrm{H}, \mathrm{OCH}_{3}\right), 3.85\left(\mathrm{~s}, 3 \mathrm{H}, \mathrm{OCH}_{3}\right), 4.91$ (d, J=5.0 Hz, 1H, H-12b), 4.99 $(\mathrm{d}, J=9.4 \mathrm{~Hz}, 1 \mathrm{H}, \mathrm{H}-3) ; 6.68$ (br s, $1 \mathrm{H}, \mathrm{NH}), 6.82-7.90\left(\mathrm{~m}, 8 \mathrm{H}, \mathrm{CH}_{\text {arom }}\right) ;{ }^{13} \mathrm{C} \mathrm{NMR}\left(\mathrm{CDCl}_{3}\right): \delta$ $15.92\left(\mathrm{CH}_{3}\right), 29.72\left(\mathrm{CH}_{\mathrm{Ad}}\right), 37.10\left(\mathrm{C}_{\mathrm{Ad}}\right), 39.88\left(\mathrm{CH}_{2} \mathrm{Ad}\right), 39.97\left(\mathrm{CH}_{2} \mathrm{Ad}\right), 51.86(\mathrm{C}-2), 53.59(\mathrm{C}-$ 1), $54.65\left(\mathrm{OCH}_{3}\right), 56.46(\mathrm{CH}-\mathrm{N}), 65.32(\mathrm{C}-12 \mathrm{~b}), 69.27(\mathrm{C}-3), 114.99-145.82\left(\mathrm{CH}_{\text {arom }}\right.$ and $\left.\mathrm{C}_{\text {arom }}\right)$, 167.08, 171.41, 173.30 (3 C=O); EI-MS: m/z (\%) $542\left(\mathrm{M}^{+}, 1\right), 325$ (1), 374 (1), 304 (2), 251 (42), 219 (10), 194 (29), 180 (100), 135 (22). Anal. Calcd. for $\mathrm{C}_{33} \mathrm{H}_{38} \mathrm{~N}_{2} \mathrm{O}_{5}$ (542.67): C, 73.04; H, 7.06; N, 5.16. Found: C, 72.92; H, 7.01; N, 4.93 .

Dimethyl $\left(1 R^{*}, 2 R^{*}, 3 S^{*}, 12 \mathrm{~b} R^{*}\right)-3$-(tert-butylaminocarbonyl)-1,2,3,12b-tetrahydropyrrolo[1,2-f]phenanthridine-1,2-dicarboxylate (6cb). Yellowish crystals (from chloroform /ether), mp 203-205 ${ }^{\circ} \mathrm{C} ; \mathrm{R}_{f}=0.67$ (silica; dichloromethane/ethyl acetate 5:1); IR (KBr): $3320(\mathrm{~N}-\mathrm{H})$, $3035\left(\mathrm{C}-\mathrm{H}_{\text {arom }}\right), 2928,2862,1732(\mathrm{C}=\mathrm{O}), 1676(\mathrm{C}=\mathrm{O}), 1528,1260,1149,1167,741 \mathrm{~cm}^{-1} ;{ }^{1} \mathrm{H}$ NMR $\left(\mathrm{CDCl}_{3}\right): \delta 1.40\left(\mathrm{~s}, 9 \mathrm{H}, 3 \mathrm{CH}_{3}\right), 3.43(\mathrm{dd}, J=9.3 \mathrm{~Hz}, J=9.3 \mathrm{~Hz}, 1 \mathrm{H}, \mathrm{H}-2), 3.72(\mathrm{~s}, 3 \mathrm{H}$, $\left.\mathrm{OCH}_{3}\right), 3.80(\mathrm{dd}, J=9.3 \mathrm{~Hz}, J=5.2 \mathrm{~Hz}, 1 \mathrm{H}, \mathrm{H}-1), 3.82\left(\mathrm{~s}, 3 \mathrm{H}, \mathrm{OCH}_{3}\right), 4.83(\mathrm{~d}, J=5.3 \mathrm{~Hz}, 1 \mathrm{H}$,

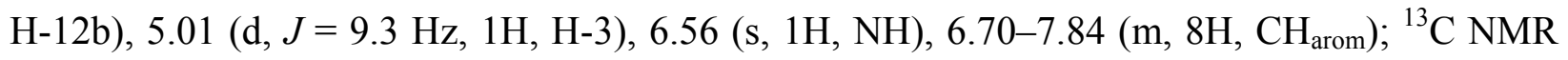
$\left(\mathrm{CDCl}_{3}\right): \delta 28.77\left(\mathrm{CH}_{3}\right), 50.49(\mathrm{C}-2), 51.34[\mathrm{C}-\mathrm{NH}], 52.80\left(\mathrm{OCH}_{3}\right), 53.72\left(\mathrm{OCH}_{3}\right), 55.25(\mathrm{C}-1)$, 64.13 (C-12b), 68.63 (C-3), 113.61-143.07 ( $\mathrm{CH}_{\text {arom }}$ and $\left.\mathrm{C}_{\text {arom }}\right), 169.91,171.94,172.26(3 \mathrm{C}=\mathrm{O})$; EI-MS: $m / z(\%) 437\left(\mathrm{M}^{+}, 5\right), 435$ (4), 405 (2), 336 (7), 304 (15), 276 (28), 251 (100), 219 (41), 217 (35), 193 (15), 180 (15), 57 (8), 41 (3). Anal. Calcd. for $\mathrm{C}_{25} \mathrm{H}_{28} \mathrm{~N}_{2} \mathrm{O}_{5}$ (436.50): C, 68.79; H, 6.47 ; N, 6.42. Found: C, 68.59; H, 6.52; N, 6.22.

Dimethyl $\quad\left(1 R^{*}, 2 R^{*}, 3 R^{*}, 12 \mathrm{~b} S^{*}\right)-3$-(tert-butylaminocarbonyl)-1,2,3,12b-tetrahydropyrrolo[1,2-f]phenanthridine-1,2-dicarboxylate (7cb). Yellow crystals (from chloroform/ether), mp 213-215 ${ }^{\circ} \mathrm{C} ; \mathrm{R}_{f}=0.52$ (silica; dichloromethane/ethyl acetate 5:1); IR (KBr): $3385.6(\mathrm{~N}-\mathrm{H}), 3039$ $\left(\mathrm{C}-\mathrm{H}_{\text {arom }}\right), 2929,2865,1731(\mathrm{C}=\mathrm{O}), 1646(\mathrm{C}=\mathrm{O}), 1526,1450,1168,741 \mathrm{~cm}^{-1}$; ${ }^{1} \mathrm{H}$ NMR $\left(\mathrm{CDCl}_{3}\right): \delta 1.39\left(\mathrm{~s}, 9 \mathrm{H}, 3 \mathrm{CH}_{3}\right), 3.39(\mathrm{~d}, J=5.7 \mathrm{~Hz}, 1 \mathrm{H}, \mathrm{H}-2), 3.75\left(\mathrm{~s}, 3 \mathrm{H}, \mathrm{OCH}_{3}\right), 3.76(\mathrm{~d}, J=$ $9.2 \mathrm{~Hz}, 1 \mathrm{H}, \mathrm{H}-1), 3.82\left(\mathrm{~s}, 3 \mathrm{H}, \mathrm{OCH}_{3}\right), 4.75$ (d, $\left.J=9.4 \mathrm{~Hz}, 1 \mathrm{H}, \mathrm{H}-12 \mathrm{~b}\right), 5.71$ (d, $J=5.8 \mathrm{~Hz}, 1 \mathrm{H}$, $\mathrm{H}-3), 6.68(\mathrm{~s}, 1 \mathrm{H}, \mathrm{NH}), 6.54-7.81\left(\mathrm{~m}, 8 \mathrm{H}, \mathrm{CH}_{\text {arom }}\right) ;{ }^{13} \mathrm{C} \mathrm{NMR}\left(\mathrm{CDCl}_{3}\right): \delta 28.58\left(\mathrm{CH}_{3}\right), 46.08(\mathrm{C}-$ 2), $51.08(\mathrm{C}-\mathrm{NH}), 55.78$ (C-1), $63.30(\mathrm{C}-12 \mathrm{~b}), 71.34(\mathrm{C}-3), 112.65-144.42\left(\mathrm{CH}_{\text {arom }}\right.$ and $\left.\mathrm{C}_{\text {arom }}\right)$, 169.33, 171.16, 172.91 (3 C=O); EI-MS: m/z (\%) $437\left(\mathrm{M}^{+}, 5\right), 435$ (4), 405 (2), 336 (7), 304 (15), 276 (28), 251 (100), 219 (41), 217 (35), 193 (15), 180 (15), 57 (8), 41 (3). Anal. Calcd. for $\mathrm{C}_{25} \mathrm{H}_{28} \mathrm{~N}_{2} \mathrm{O}_{5}$ (436.50): C, 68.79; H, 6.47; N, 6.42. Found: C, 68.58; H, 6.52; N, 6.63.

Dimethyl $\left(1 R^{*}, 2 R^{*}, 3 S^{*}, 12 \mathrm{~b} R^{*}\right)-3$-(ethylaminocarbonyl)-1,2,3,12b-tetrahydropyrrolo-[1,2flphenanthridine-1,2-dicarboxylate (6db). Pale red crystals (from chloroform/ether), mp 228$230{ }^{\circ} \mathrm{C} ; \mathrm{R}_{f}=0.75$ (silica; ethyl acetate); IR (KBr): $3424(\mathrm{NH}), 3075,2979,2946,1778(\mathrm{C}=\mathrm{O})$, $1704(\mathrm{C}=\mathrm{O}), 1541,1443,1360,1204,1092,744 \mathrm{~cm}^{-1} ;{ }^{1} \mathrm{H} \mathrm{NMR}\left(\mathrm{CDCl}_{3}\right): \delta 1.11\left(\mathrm{t}, 3 \mathrm{H}, \mathrm{CH}_{3}\right)$, $3.52\left(\mathrm{q}, 2 \mathrm{H}, \mathrm{CH}_{2} \mathrm{~N}\right), 3.23(\mathrm{dd}, J=9.4 \mathrm{~Hz}, J=9.4 \mathrm{~Hz}, 1 \mathrm{H}, \mathrm{H}-2), 3.65$ (dd, $J=9.3 \mathrm{~Hz}, J=5.2 \mathrm{~Hz}$, $1 \mathrm{H}, \mathrm{H}-1), 3.75$ (s, 6H, OCH 3$), 4.91$ (d, $J=5.3 \mathrm{~Hz}, 1 \mathrm{H}, \mathrm{H}-12 \mathrm{~b}), 4.99$ (d, $J=9.5 \mathrm{~Hz}, 1 \mathrm{H}, \mathrm{H}-3)$, $6.68(\mathrm{~s}, 1 \mathrm{H}, \mathrm{NH}), 7.06-7.98\left(\mathrm{~m}, 8 \mathrm{H}, \mathrm{CH}_{\text {arom }}\right) ;{ }^{13} \mathrm{C} \mathrm{NMR}\left(\mathrm{CDCl}_{3}\right): \delta 12.95\left(\mathrm{CH}_{3}\right), 34.71\left(\mathrm{CH}_{2} \mathrm{~N}\right)$, $48.77(\mathrm{C}-2), 51.71\left(\mathrm{OCH}_{3}\right), 52.86\left(\mathrm{OCH}_{3}\right), 53.59$ (C-1), $56.46(\mathrm{C}-12 \mathrm{~b}), 69.27$ (C-3), 114.99- 
$151.19\left(\mathrm{CH}_{\text {arom }}\right.$ and $\left.\mathrm{C}_{\text {arom }}\right), 165.60,173.11,175.48(3 \mathrm{C}=\mathrm{O})$; EI-MS: $m / z(\%) 408\left(\mathrm{M}^{+}, 1\right), 376$ (70), 343 (22), 316 (28), 276 (51), 244 (100), 217 (39), 191 (20), 122 (15), 108 (29), 94 (28), 56 (5), 44 (3). Anal. Calcd. for $\mathrm{C}_{23} \mathrm{H}_{24} \mathrm{~N}_{2} \mathrm{O}_{5}$ (408.45): C, 67.63; H, 5.92; N, 6.86. Found: C, 67.77; H, 5.62; N, 6.62 .

\section{Products from the reaction of salts 3a-e with dimethyl maleate 5c}

Dimethyl (2R*,3R*)-3-(1-adamantyl)aminocarbonyl-2,3-dihydropyrrolo[1,2-f]phenanthridine-1,2-dicarboxylate ${ }^{7}$ (8ac). Pale orange crystals (from chloroform/ether), mp 189-192 ${ }^{\circ} \mathrm{C}$; $\mathrm{R}_{f}$ $=0.81$ (silica; dichloromethane/ether 2:1); IR (KBr): $3342(\mathrm{~N}-\mathrm{H}), 3061\left(\mathrm{C}-\mathrm{H}_{\text {arom }}\right), 2908,2851$, $1740(\mathrm{C}=\mathrm{O}), 1678(\mathrm{C}=\mathrm{O}), 1602,1519,1444,1263,1094,755 \mathrm{~cm}^{-1} ;{ }^{1} \mathrm{H}$ NMR $\left(\mathrm{CDCl}_{3}\right): \delta 1.60-$ $1.88\left(\mathrm{~m}, 12 \mathrm{H}, 6 \mathrm{CH}_{2} \mathrm{Ad}\right) ; 1.99\left(\mathrm{~s}, 3 \mathrm{H}, 3 \mathrm{CH}_{\mathrm{Ad}}\right), 3.73\left(\mathrm{~s}, 3 \mathrm{H}, \mathrm{OCH}_{3}\right), 3.76\left(\mathrm{~s}, 3 \mathrm{H}, \mathrm{OCH}_{3}\right), 4.21(\mathrm{~d}$, $J=5.0 \mathrm{~Hz}, 1 \mathrm{H}, \mathrm{H}-2), 4.79(\mathrm{~d}, J=5.0 \mathrm{~Hz}, 1 \mathrm{H}, \mathrm{H}-3), 6.15(\mathrm{~s}, 1 \mathrm{H}, \mathrm{NH}), 6.82-8.53\left(\mathrm{~m}, 8 \mathrm{H}, \mathrm{CH}_{\text {aro }}\right)$; ${ }^{13} \mathrm{C}$ NMR $\left(\mathrm{CDCl}_{3}\right): \delta 29.25\left(\mathrm{CH}_{\mathrm{Ad}}\right), 34.22(\mathrm{C}-2), 36.01\left(\mathrm{CH}_{2 \mathrm{Ad}}\right), 41.05(\mathrm{C}-1), 41.24\left(\mathrm{CH}_{2} \mathrm{Ad}\right)$, $51.30\left(\mathrm{OCH}_{3}\right), 52.82\left(\mathrm{OCH}_{3}\right), 52.58\left(\mathrm{C}_{\mathrm{Ad}}\right), 65.52(\mathrm{C}-3), 95.27(\mathrm{C}-12 \mathrm{~b}), 113.06-137.21\left(\mathrm{CH}_{\text {arom }}\right.$ and $\left.\mathrm{C}_{\text {arom }}\right), 173.49(\mathrm{C}=\mathrm{O})$; EI-MS: $m / z(\%) 512\left(\mathrm{M}^{+}, 1\right), 386(4), 302$ (3), 251 (5), 236 (5), 210 (10), 209 (100), 178 (15), 135 (11), 91 (18), 41 (3). Anal. Calcd. for $\mathrm{C}_{31} \mathrm{H}_{32} \mathrm{~N}_{2} \mathrm{O}_{5}$ (512.60): C, 72.64; H, 6.29; N, 5.46. Found: C, 72.36; H, 6.00; N, 5.52.

Dimethyl $\left(1 R^{*}, 2 S^{*}, 3 R^{*}, 12 \mathrm{~b} R^{*}\right)-3-[(1 R S)-1-(1-a d a m a n t y l)-1-e t h y l a m i n o c a r b o n y l]-1,2,3,12 \mathrm{~b}-$ tetrahydropyrrolo[1,2-f]phenanthridine-1,2-dicarboxylate (11bc). Orange crystals (from chloroform/ether), $\mathrm{mp} 248-250{ }^{\circ} \mathrm{C} ; \mathrm{R}_{f}=0.66$ (silica; ethyl acetate); IR (KBr): $3294(\mathrm{NH}), 3061$, 2902, 2847, $1738(\mathrm{C}=\mathrm{O}), 1654(\mathrm{C}=\mathrm{O}), 1543,1439,1163,743 \mathrm{~cm}^{-1} ;{ }^{1} \mathrm{H} \mathrm{NMR}\left(\mathrm{CDCl}_{3}\right): \delta 1.04(\mathrm{~d}$, $\left.J=6.9 \mathrm{~Hz}, 3 \mathrm{H}, \mathrm{CH}_{3}\right), 1.36(\mathrm{q}, J=6.9 \mathrm{~Hz}, 1 \mathrm{H}, \mathrm{HC}-\mathrm{N}), 1.53-1.62\left(\mathrm{~m}, 12 \mathrm{H}, 6 \mathrm{CH}_{2} \mathrm{Ad}\right), 1.99$ (s, $3 \mathrm{H}, 3 \mathrm{CH}_{\mathrm{Ad}}$ ), 3.41 (d, $\left.J=9.4 \mathrm{~Hz}, 1 \mathrm{H}, \mathrm{H}-2\right), 3.45$ (d, $\left.J=9.4 \mathrm{~Hz}, 1 \mathrm{H}, \mathrm{H}-2\right), 4.25$ (d, $J=3.95 \mathrm{~Hz}$, $1 \mathrm{H}, \mathrm{H}-1), 4.29$ (d, $J=3.75 \mathrm{~Hz}, 1 \mathrm{H}, \mathrm{H}-1), 4.92$ (d, $J=5.0 \mathrm{~Hz}, 1 \mathrm{H}, \mathrm{H}-12 \mathrm{~b}) ; 4.95$ (d, $J=4.7 \mathrm{~Hz}$, 1H, H-12b), 5.00 (d, $J=9.3 \mathrm{~Hz}, 1 \mathrm{H}, \mathrm{H}-3), 5.05$ (d, $J=9.5 \mathrm{~Hz}, 1 \mathrm{H}, \mathrm{H}-3), 6.59$ (br, 1H, N-H), 6.70-7.80 (m, 8H, $\left.\mathrm{CH}_{\text {arom }}\right) ;{ }^{13} \mathrm{C} \mathrm{NMR}\left(\mathrm{CDCl}_{3}\right): \delta 15.92\left(\mathrm{CH}_{3}\right), 29.72\left(\mathrm{CH}_{\mathrm{Ad}}\right), 37.10\left(\mathrm{C}_{\mathrm{Ad}}\right), 39.88$ $\left(\mathrm{CH}_{2} \mathrm{Ad}\right), 39.97\left(\mathrm{CH}_{2} \mathrm{Ad}\right), 51.86(\mathrm{C}-2), 52.12\left(\mathrm{OCH}_{3}\right), 52.42\left(\mathrm{OCH}_{3}\right), 52.22(\mathrm{C}-2), 53.59(\mathrm{C}-1)$, 53.72 (C-1), 56.46 (C-12b), 56.61 (C-12b), $65.32(\mathrm{CH}-\mathrm{N}), 65.90(\mathrm{CH}-\mathrm{N}), 69.27$ (C-3), 69.32 (C3), 114.99-145.82 ( $\mathrm{CH}_{\text {arom }}$ and $\left.\mathrm{C}_{\text {arom }}\right), 166.80,167.08,171.21,171.41,173.30$ (3 C=O); EI-MS: m/z (\%) $542\left(\mathrm{M}^{+}, 1\right), 325$ (1), 374 (1), 304 (2), 251 (42), 219 (10), 194 (29), 180 (100), 135 (22). Anal. Calcd. for $\mathrm{C}_{33} \mathrm{H}_{38} \mathrm{~N}_{2} \mathrm{O}_{5}$ (542.67): C, 73.04; H, 7.06; N, 5.16. Found: C, 72.92; H, 7.16; N, 5.28 .

Dimethyl $\left(2 R, * 3 R^{*}\right)$-3-(tert-butyl)aminocarbonyl-2,3-dihydropyrrolo[1,2-f]phenanthridine1,2-dicarboxylate (8cc). Yellow crystals (from chloroform/ether), mp 169.2-171.1 ${ }^{\circ} \mathrm{C} ; \mathrm{R}_{f}=0.68$ (silica; ethyl acetate/ dichloromethane 1:5); IR (KBr): $3368(\mathrm{~N}-\mathrm{H}), 3075\left(\mathrm{C}-\mathrm{H}_{\text {arom }}\right), 2965,1738$ $(\mathrm{C}=\mathrm{O}), 1671(\mathrm{C}=\mathrm{O}), 1528,1443,1361,1290,1168,1098,753 \mathrm{~cm}^{-1} ;{ }^{1} \mathrm{H} \mathrm{NMR}\left(\mathrm{CDCl}_{3}\right): \delta 1.40$ $\left(\mathrm{s}, 9 \mathrm{H}, 3 \mathrm{CH}_{3}\right), 3.75\left(\mathrm{~s}, 3 \mathrm{H}, \mathrm{OCH}_{3}\right), 3.78\left(\mathrm{~s}, 3 \mathrm{H}, \mathrm{OCH}_{3}\right), 4.23(\mathrm{~d}, J=4.97 \mathrm{~Hz}, 1 \mathrm{H}, \mathrm{H}-2), 4.85$ (d, $J=4.97 \mathrm{~Hz}, 1 \mathrm{H}, \mathrm{H}-3), 5.64(\mathrm{~s}, 1 \mathrm{H}, \mathrm{NH}), 6.45-8.13\left(\mathrm{~m}, \mathrm{CH}_{\text {arom }}\right) ;{ }^{13} \mathrm{C} \mathrm{NMR}\left(\mathrm{CDCl}_{3}\right): \delta 28.44$ $\left(\mathrm{CH}_{3}\right), 51.41\left(\mathrm{OCH}_{3}\right), 52.22(\mathrm{C}-2), 52.65\left(\mathrm{OCH}_{3}\right), 54.55(\mathrm{C}-\mathrm{N}), 65.53(\mathrm{C}-3), 95.34(\mathrm{C}-1)$, 
111.59-136.25 ( $\mathrm{CH}_{\text {arom }}$ and $\left.\mathrm{C}_{\text {arom }}\right), 169.91,171.94,172.26(3 \mathrm{C}=\mathrm{O}), \mathrm{EI}-\mathrm{MS}: \mathrm{m} / z(\%) 434\left(\mathrm{M}^{+}\right.$, 1), 308 (11), 276 (2), 236 (9), 210 (12), 209 (100), 180 (38), 178 (50), 152 (22), 57 (12), 32 (9), 28 (31). Anal. Calcd. for $\mathrm{C}_{25} \mathrm{H}_{26} \mathrm{~N}_{2} \mathrm{O}_{5}$ (434.49): C, 69.11; H, 6.03; N, 6.45. Found: C, 68.92; H, $6.12 ; \mathrm{N}, 6.45$.

\section{Products from the reaction of salt $3 \mathrm{~d}$ with dimethyl maleate $5 \mathrm{c}$}

Dimethyl $\left(2 R, * 3 R^{*}\right)-3$-(ethylaminocarbonyl)-2,3-dihydropyrrolo[1,2-flphenanthridine-1,2dicarboxylate (8dc). Pale yellow crystals (from chloroform/ether), $\mathrm{mp}>350{ }^{\circ} \mathrm{C} ; \mathrm{R}_{f}=0.35$ (silica; ether/dichloromethane 1:5); IR (KBr): $3359(\mathrm{NH}), 3070,2947,1742(\mathrm{C}=\mathrm{O}), 1665(\mathrm{C}=\mathrm{O})$, 1524, 1443, 1299, 1171, 1094, $752 \mathrm{~cm}^{-1} ;{ }^{1} \mathrm{H}$ NMR $\left(\mathrm{CDCl}_{3}\right): \delta 1.02\left(\mathrm{t}, 3 \mathrm{H}, \mathrm{CH}_{3}\right), 3.25(\mathrm{q}$, $\left.2 \mathrm{H}, \mathrm{CH}_{2} \mathrm{~N}\right), 3.73\left(\mathrm{~s}, 3 \mathrm{H}, \mathrm{OCH}_{3}\right), 3.77\left(\mathrm{~s}, 3 \mathrm{H}, \mathrm{OCH}_{3}\right), 4.23(\mathrm{~d}, J=4.39 \mathrm{~Hz}, 1 \mathrm{H}, \mathrm{H}-2), 4.99(\mathrm{~d}, J=$ $4.34 \mathrm{~Hz}, 1 \mathrm{H}, \mathrm{H}-3), 5.59$ (s, $1 \mathrm{H}, \mathrm{NH}), 6.58-9.64\left(\mathrm{~m}, \mathrm{CH}_{\text {arom }}\right) ;{ }^{13} \mathrm{C} \mathrm{NMR}\left(\mathrm{CDCl}_{3}\right): \delta 14.76\left(\mathrm{CH}_{3}\right)$, $34.81\left(\mathrm{CH}_{2} \mathrm{~N}\right), 51.32(\mathrm{C}-2), 51.62\left(\mathrm{OCH}_{3}\right), 52.78\left(\mathrm{OCH}_{3}\right), 65.26(\mathrm{C}-3), 95.76(\mathrm{C}-1), 113.34-$ $150.89\left(\mathrm{CH}_{\text {arom }}\right.$ and $\left.\mathrm{C}_{\text {arom }}\right), 165.92,168.77,173.62(3 \mathrm{C}=\mathrm{O})$; EI-MS: $m / z(\%) 408\left(\mathrm{M}^{+}, 1\right), 376$ (70), 343 (22), 316 (28), 276 (51), 244 (100), 217 (39), 191 (20), 122 (15), 108 (29), 94 (28), 56 (5), 44 (3). Anal. Calcd. for $\mathrm{C}_{23} \mathrm{H}_{22} \mathrm{~N}_{2} \mathrm{O}_{5}$ (406.43): C, 67.97; H, 5.46; N, 6.89. Found: C, 67.87; H, 5.26; N, 6.64 .

Dimethyl $\quad\left(2 R, * 3 R^{*}\right)-3-($ benzylaminocarbonyl)-2,3-dihydropyrrolo[1,2-f]phenanthridine1,2-dicarboxylate (8ec). White crystals (from chloroform/ether), $\mathrm{mp}>350{ }^{\circ} \mathrm{C} ; \mathrm{R}_{f}=0.75$ ( silica; ethyl acetate/petroleum ether 1:3); IR (KBr): $3325(\mathrm{NH}), 3066\left(\mathrm{C}-\mathrm{H}_{\text {arom }}\right), 3032,2889,1742$ $(\mathrm{C}=\mathrm{O}), 1665(\mathrm{C}=\mathrm{O}), 1524,1443,1299,1171,1094,752 \mathrm{~cm}^{-1} ;{ }^{1} \mathrm{H}$ NMR $\left(\mathrm{CDCl}_{3}\right)$ : $\delta 3.73(\mathrm{~s}, 3 \mathrm{H}$, $\left.\mathrm{OCH}_{3}\right), 3.77\left(\mathrm{~s}, 3 \mathrm{H}, \mathrm{OCH}_{3}\right), 4.23(\mathrm{~d}, J=3.59 \mathrm{~Hz}, 1 \mathrm{H}, \mathrm{H}-2), 4.48\left(\mathrm{~d}, 2 \mathrm{H}, \mathrm{CH}_{2} \mathrm{~N}\right), 4.99(\mathrm{~d}, J=$ $3.68 \mathrm{~Hz}, 1 \mathrm{H}, \mathrm{H}-3), 5.62(\mathrm{~s}, 1 \mathrm{H}, \mathrm{NH}), 6.58-9.64\left(\mathrm{~m}, \mathrm{CH}_{\text {arom }}\right) ;{ }^{13} \mathrm{C} \mathrm{NMR}\left(\mathrm{CDCl}_{3}\right): \delta 43.61$ $\left(\mathrm{CH}_{2} \mathrm{~N}\right), 51.32(\mathrm{C}-2), 52.84\left(\mathrm{OCH}_{3}\right), 54.12\left(\mathrm{OCH}_{3}\right), 65.26(\mathrm{C}-3), 95.76(\mathrm{C}-1), 113.34-150.89$ $\left(\mathrm{CH}_{\text {arom }}\right.$ and $\left.\mathrm{C}_{\text {arom }}\right), 165.92,168.77,173.62(3 \mathrm{C}=\mathrm{O})$; EI-MS: $m / z(\%) 471\left(\mathrm{M}^{+}, 1\right), 376(50), 343$ (12), 316 (28), 276 (51), 244 (100), 217 (35), 191 (21), 121 (15), 108 (29), 94 (28), 56 (5), 44 (3). Anal. Calcd. for $\mathrm{C}_{28} \mathrm{H}_{24} \mathrm{~N}_{2} \mathrm{O}_{5}$ (468.50): C, 71.78; H, 5.16; N, 5.98. Found: C, 71.48; H, 4.92; N, 5.62 .

\section{Acknowledgements}

Financial support from Ministry of Education (J07/98:143100011) and Ministry of Industry and Trade (Grant PZ-CH/25) is gratefully acknowledged.

\section{References and Notes}

1. Potáček, M.; Topinka, T.; Dostál, J.; Humpa, J. Collect. Czech. Chem. Commun. 1995, 60, 
1191.

2. Potáček, M.; Topinka, T.; Dostál, J.; Marek, J. Collect. Czech. Chem. Commun. 1994, 59, 2641.

3. Dostál, J.; Potáček, M.; Humpa, O.; Marek, J. Bull. Soc. Chim. Belg. 1994, 103, 343.

4. Topinka, T. M .S. Thesis, Masaryk University Brno, 1995.

5. Dostál, J. Ph. D. Thesis, Masaryk University Brno, 1993.

6. Trávníček, M. M.S. Thesis, Masaryk University Brno, 1998.

7. Trávníček, M.; Pospíšil, J.; Potáček, M. Collect. Czech. Chem. Commun. 1999, 64, 1993.

8. Shamma, M. Isoquinoline Alkaloids, Academic Press: New York, 1983.

9. Shamma, M.; Moniot, J. L. Isoquinoline Alkaloids Research, Plenum: New York, 1978; 1972-1977.

10. Preininger, V. The Alkaloids, Academic Press: New York, 1975; Vol. 15, p 207.

11. Šimánek, V. The Alkaloids, Academic Press: New York, 1985; Vol. 26, p 185.

12. Preininger, V. The Alkaloids, Academic Press: New York, 1986; Vol. 29, p 1.

13. Wishnok, J. S. J. Chem. Educ. 1973, 50, 780.

14. Davies, W. L.; Grunert, R. R.; Haff, R. F. Science 1964, 144, 862.

15. Plánička, K. Research Report No. 1470, 1979, Research Institute for Pharmacy and Biochemistry, Prague.

16. Woller, P. B.; Cromwell, N. H. J. Org.Chem. 1970, 35, 888.

17. Karplus, M. J. Chem. Phys. 1959, 30, 11. 